\title{
A Leap-Frog Finite Difference Method for Strongly Coupled System from Sweat Transport in Porous Textile Media
}

\author{
Qian Zhang ${ }^{1,2}$ and Chao Huang ${ }^{1,3}$ \\ ${ }^{1}$ College of Mathematics and Statistics, Shenzhen University, China \\ ${ }^{2}$ Shenzhen Key Laboratory of Advanced Machine Learning and Applications, China \\ ${ }^{3}$ Guangdong Key Laboratory of Intelligent Information Processing, China
}

Correspondence should be addressed to Qian Zhang; mazhangq@szu.edu.cn

Received 25 March 2019; Accepted 15 May 2019; Published 12 June 2019

Academic Editor: Tepper L. Gill

Copyright (C) 2019 Qian Zhang and Chao Huang. This is an open access article distributed under the Creative Commons Attribution License, which permits unrestricted use, distribution, and reproduction in any medium, provided the original work is properly cited.

In this paper, we present an uncoupled leap-frog finite difference method for the system of equations arising from sweat transport through porous textile media. Based on physical mechanisms, the sweat transport can be viewed as the multicomponent flow that coupled the heat and moisture transfer, such that the system is nonlinear and strongly coupled. The leap-frog method is proposed to solve this system, with the second order accuracy in both spatial and temporal directions. We prove the existence and uniqueness of the solution to the system with optimal error estimates in the discrete $L^{2}$ norm. Numerical simulations are presented and analyzed, respectively.

\section{Introduction}

Single/multicomponent flow in porous textile media attracted considerable attention in the last several decades. See [1-4] for the single-component models and [5-9] for the multicomponent models. In this paper, we study the multicomponent sweat transport coupled with vapor and heat in porous textile media. In [10], Ye et al. proposed a quasi-steady-state single-component model which consists of a steady-state air equation and dynamic state equations for other components. Under certain conditions, the multicomponent model reduces to a new single-component model, and the physical process can be viewed as sweat transport (vapor and heat flow) governed by the conservation of mass and energy:

$$
\begin{aligned}
& \frac{\partial}{\partial t}(\epsilon C)+\frac{\partial}{\partial x}\left(u_{g} \epsilon C\right)=-\Gamma_{c e}, \\
& \frac{\partial}{\partial t}\left(C_{v t} T\right)+\frac{\partial}{\partial x}\left(\epsilon u_{g} C_{m g} C T\right)-\frac{\partial}{\partial x}\left(\kappa \frac{\partial T}{\partial x}\right) \\
& \quad=\lambda M_{w} \Gamma_{c e},
\end{aligned}
$$

where $\epsilon$ is the porosity of the media, $C$ is the vapor concentration, $T$ is the temperature, $\kappa$ is the thermal conductivity, $\lambda$ is the latent heat of evaporation/condensation, and $M_{w}$ is the molecular weight of water. The effective volumetric heat capacity $C_{v t}$ is defined by

$$
C_{v t}=\epsilon C_{m g} C+(1-\epsilon) C_{v f},
$$

where $C_{m g}$ is the molar heat capacity and $C_{v f}$ is the volumetric heat capacity of fiber.

By Darcy's law, the gas velocity $u_{g}$ is defined as

$$
u_{g}=-\frac{k}{\mu} \frac{\partial P}{\partial x}
$$

where $k$ is the permeability and $\mu$ is the dynamic viscosity, which usually is density-dependent for the compressible flow. Here we choose a linear form of $\mu:=\nu C$, where $\nu$ is a certain constant.

By the Hertz-Knudsen equation [11], the phase change rate $\Gamma_{c e}$ is defined as

$$
\Gamma_{\mathrm{ce}}=\beta_{\Gamma}\left(\frac{P-P_{\mathrm{sat}}(T)}{\sqrt{T}}\right),
$$


where $\beta_{\Gamma}$ is a positive constant, the saturation pressure $P_{\text {sat }}$ is determined from experimental measurements [12], and the pressure $P$ is given by $P=R C T$, where $R$ is the universal gas constant.

With nondimensionalization, the sweat transport process (1)-(2) can be described by the following system:

$$
\begin{gathered}
c_{t}-\left((c \theta)_{x}\right)_{x}=-\Gamma(c, \theta), \\
0<x<L, t>0, \\
(c \theta)_{t}+(\sigma \theta)_{t}-\left((c \theta)_{x} \theta\right)_{x}-\kappa \theta_{x x}=\lambda \Gamma(c, \theta), \\
0<x<L, t>0,
\end{gathered}
$$

where $(\cdot)_{x}=\partial / \partial x,(\cdot)_{t}=\partial / \partial t, \Gamma(c, \theta)=c \sqrt{\theta}-p_{s}(\theta)$, and $p_{s}(\theta) \sim P_{s a t}(\theta) / \sqrt{\theta}$ is a smooth and increasing function satisfying $p_{s}(0)=0$.

Since the right boundary is exposed to environment and the left boundary is connected to the body, we consider commonly used Robin type boundary conditions

$$
\begin{aligned}
(c \theta)_{x}=\alpha_{1}\left(c-\mu_{1}\right), & x=0,0<t \leq T, \\
(c \theta)_{x}=\alpha_{2}\left(\mu_{2}-c\right), & x=L, 0<t \leq T, \\
\theta_{x}=\beta_{1}\left(\theta-\nu_{1}\right), & x=0,0<t \leq T, \\
\theta_{x}=\beta_{2}\left(\nu_{2}-\theta\right), & x=L, 0<t \leq T,
\end{aligned}
$$

and the initial conditions

$$
\begin{aligned}
& c(x, 0)=c_{0}(x), \\
& \theta(x, 0)=\theta_{0}(x), \\
& \quad 0 \leq x \leq L .
\end{aligned}
$$

Physically, parameters $\alpha_{i}, \beta_{i}, \mu_{i}, v_{i}, i=1,2$, and $\sigma$ are nonnegative constants $[1,2,6]$. We define initial condition parameters $c_{0}(x) \geq \underline{c}, \theta_{0}(x) \geq \underline{\theta}$ with $\underline{c}$ and $\underline{\theta}$ being positive constants.

Due to the strong nonlinearity and the coupling of the system, both theoretical and numerical analyses of the system are difficult. Numerical analysis for some related systems of parabolic/elliptic equations can be found in [13-20]. Existence and uniqueness of a classical solution for a steadystate model was given in [10]. Existence of a weak solution for the corresponding dynamic models was given in $[21,22]$. Positivity of temperature and nonnegativity of vapor density were also proved here. Recently, a finite difference method second-order in space and first-order in time for the system (6)-(12) was presented in [23], where the backward semiimplicit Euler scheme is applied in the temporal direction and central finite difference approximations are used in the spatial direction. In [23], authors presented optimal error estimates under the assumption that the step size $\tau$ and $h$ are smaller than a positive constant.

In this paper, we propose an uncoupled leap-frog finite difference method for the system (6)-(12) with second-order accuracy in both spatial and temporal directions. We prove the existence and uniqueness of a solution to the finite difference system with optimal error estimates in the discrete $L^{2}$ norm, under the condition that the mesh size $\tau$ and $h$ are smaller than a positive constant which depends solely upon the physical parameters involved in the equations. Due to the strong nonlinearity and the coupling of equations, the method presented in [23] does not apply to the leap-frog scheme directly. One of the difficulties is to show convergence of the numerical solution without restriction on the grid ratio. In this paper, we assume that the solution $(c(x, t), \theta(x, t))$ to the system (6)-(12) satisfies that

$$
\begin{aligned}
& c_{\min } \leq c(x, t) \leq c_{\max }, \\
& \theta_{\min } \leq \theta(x, t) \leq \theta_{\max }
\end{aligned}
$$

for some positive constants $c_{\min }, c_{\max }, \theta_{\max }$, and $\theta_{\min }$.

The manuscript is organized as follows: in Section 2, we present an uncoupled leap-frog finite difference method for the nonlinear sweat transport system. In Section 3, we prove the existence and uniqueness of the solution to the sweat transport system with the optimal error estimate in the discrete $L^{2}$ norm. Numerical results will be presented in Section 4 to support our theoretical results.

\section{The Leap-Frog Finite Difference Scheme}

For convenience of calculations, we add the equation (6) times $\theta$ into the equation (7); thus the governing system (6)(7) can be modified as

$$
\begin{gathered}
c_{t}-\left((c \theta)_{x}\right)_{x}=-\Gamma(c, \theta), \\
0<x<L, t>0, \\
(c+\sigma) \theta_{t}-(c \theta)_{x} \theta_{x}-\kappa \theta_{x x}=(\lambda+\theta) \Gamma(c, \theta), \\
0<x<L, t>0 .
\end{gathered}
$$

Due to the practical interest in a long time period, say 8-24 hours, we present an uncoupled leap-frog finite difference scheme in the temporal direction and the central finite difference (volume) scheme in the spatial direction for the above system with the initial/boundary conditions (8)-(12).

Let $\mathscr{T}$ be a positive number, let $\Omega_{h}=\left\{x_{i} \mid x_{i}=\right.$ $i h, 0 \leq i \leq M\}$ be a uniform partition in $[0, L]$, and let $\Omega_{\tau}=\left\{t_{n} \mid t_{n}=n \tau, 0 \leq n \leq N\right\}$ be a uniform partition in $[0, \mathscr{T}]$, where $h=L / M$ and $\tau=\mathscr{T} / N$ are the step size in the spatial and temporal directions, respectively. Denote $x_{i+1 / 2}=\left(x_{i}+x_{i+1}\right) / 2$ in the spatial cell and let $\left\{v_{i}^{n} \mid 0 \leq i \leq\right.$ $M, 0 \leq n \leq N\}$ be a mesh function defined on $\Omega_{h \tau}$, where $\Omega_{h \tau}=\Omega_{h} \times \Omega_{\tau}$. Some notations are introduced below:

$$
\begin{aligned}
\nabla_{t} v_{i}^{n} & =\frac{1}{2 \tau}\left(v_{i}^{n+1}-v_{i}^{n-1}\right), \\
v_{i}^{\widehat{n}} & =\frac{1}{2}\left(v_{i}^{n+1}+v_{i}^{n-1}\right), \\
v_{i+1 / 2}^{\widehat{n}} & =\frac{1}{2}\left(v_{i+1}^{n+1}+v_{i}^{n-1}\right), \\
v_{i+1 / 2}^{n} & =\frac{1}{2}\left(v_{i}^{n}+v_{i+1}^{n}\right),
\end{aligned}
$$




$$
\begin{aligned}
\delta_{x} v_{i+1 / 2}^{n} & =\frac{1}{h}\left(v_{i+1}^{n}-v_{i}^{n}\right), \\
\delta_{x}^{*} v_{i}^{\widehat{n}} & =\frac{1}{h}\left(v_{i+1 / 2}^{\widehat{n}}-v_{i-1 / 2}^{\widehat{n}}\right), \\
\delta_{x} v_{i+1 / 2}^{\widehat{n}} & =\frac{1}{2}\left(\delta_{x} v_{i+1 / 2}^{n+1}+\delta_{x} v_{i+1 / 2}^{n-1}\right), \\
\delta_{x}^{2} v_{i+1 / 2}^{\widehat{n}} & =\frac{1}{h}\left(\delta_{x} v_{i+1 / 2}^{\widehat{n}}-\delta_{x} v_{i-1 / 2}^{\widehat{n}}\right),
\end{aligned}
$$

from which

$$
\left(\delta_{x} v_{i+1 / 2}^{m}\right) v_{i+1 / 2}^{n}+v_{i+1 / 2}^{m} \delta_{x} v_{i+1 / 2}^{n}=\delta_{x}\left(v_{i+1 / 2}^{m} v_{i+1 / 2}^{n}\right) .
$$

The discrete system is defined by

$$
\begin{aligned}
& \nabla_{t} c_{i}^{n}-\frac{1}{h}\left[\left(c_{i+1 / 2}^{n} \delta_{x} \theta_{i+1 / 2}^{n}+\theta_{i+1 / 2}^{n} \delta_{x} c_{i+1 / 2}^{\hat{n}}\right)\right. \\
& \left.-\left(c_{i-1 / 2}^{n} \delta_{x} \theta_{i-1 / 2}^{n}+\theta_{i-1 / 2}^{n} \delta_{x} c_{i-1 / 2}^{\widehat{n}}\right)\right]=-\Gamma\left(c_{i}^{n}, \theta_{i}^{n}\right), \\
& 1 \leq i \leq M-1, \\
& \nabla_{t} c_{0}^{n}-\frac{2}{h}\left[\left(c_{1 / 2}^{n} \delta_{x} \theta_{1 / 2}^{n}+\theta_{1 / 2}^{n} \delta_{x} c_{1 / 2}^{\widehat{n}}\right)-\alpha_{1}\left(c_{0}^{\widehat{n}}-\mu_{1}\right)\right] \\
& =-\Gamma\left(c_{0}^{n}, \theta_{0}^{n}\right) \text {, } \\
& \nabla_{t} c_{M}^{n}-\frac{2}{h}\left[\alpha_{2}\left(\mu_{2}-c_{M}^{\widehat{n}}\right)\right. \\
& \left.-\left(c_{M-1 / 2}^{n} \delta_{x} \theta_{M-1 / 2}^{n}+\theta_{M-1 / 2}^{n} \delta_{x} c_{M-1 / 2}^{\widehat{n}}\right)\right] \\
& =-\Gamma\left(c_{M}^{n}, \theta_{M}^{n}\right) \text {, } \\
& \left(c_{i}^{\widehat{n}}+\sigma\right) \nabla_{t} \theta_{i}^{n}-\frac{1}{2}\left(u_{i-1 / 2}^{\widehat{n}} \delta_{x} \theta_{i-1 / 2}^{\widehat{n}}+u_{i+1 / 2}^{\widehat{n}} \delta_{x} \theta_{i+1 / 2}^{\widehat{n}}\right) \\
& -\frac{\kappa}{h}\left(\delta_{x} \theta_{i+1 / 2}^{\widehat{n}}-\delta_{x} \theta_{i-1 / 2}^{\widehat{n}}\right)=\left(\lambda+\theta_{i}^{n}\right) \Gamma\left(c_{i}^{n}, \theta_{i}^{n}\right), \\
& 1 \leq i \leq M-1, \\
& \left(c_{0}^{\widehat{n}}+\sigma\right) \nabla_{t} \theta_{0}^{n}-u_{1 / 2}^{\widehat{n}} \delta_{x} \theta_{1 / 2}^{\widehat{n}}-\frac{2 \kappa}{h}\left[\delta_{x} \theta_{1 / 2}^{\widehat{n}}\right. \\
& \left.-\beta_{1}\left(\theta_{0}^{\widehat{n}}-v_{1}\right)\right]=\left(\lambda+\theta_{0}^{n}\right) \Gamma\left(c_{0}^{n}, \theta_{0}^{n}\right), \\
& \left(c_{M}^{\widehat{n}}+\sigma\right) \nabla_{t} \theta_{M}^{n}-u_{M-1 / 2}^{\widehat{n}} \delta_{x} \theta_{M-1 / 2}^{\widehat{n}} \\
& -\frac{2 \kappa}{h}\left[\beta_{2}\left(\nu_{2}-\theta_{M}^{\widehat{n}}\right)-\delta_{x} \theta_{M-1 / 2}^{\widehat{n}}\right]=\left(\lambda+\theta_{M}^{n}\right) \\
& \cdot \Gamma\left(c_{M}^{n}, \theta_{M}^{n}\right), \quad 1 \leq n \leq N-1,
\end{aligned}
$$

and the discrete initial conditions

$$
\begin{aligned}
c_{i}^{0} & =c_{0}\left(x_{i}\right), \\
\theta_{i}^{0} & =\theta_{0}\left(x_{i}\right), \\
c_{i}^{1} & =c_{0}\left(x_{i}\right)+\tau c_{t}\left(x_{i}, 0\right), \\
\theta_{i}^{1} & =\theta_{0}\left(x_{i}\right)+\tau \theta_{t}\left(x_{i}, 0\right), \quad 0 \leq i \leq M,
\end{aligned}
$$

where

$$
u_{i+1 / 2}^{\widehat{n}}=c_{i+1 / 2}^{n} \delta_{x} \theta_{i+1 / 2}^{n}+\theta_{i+1 / 2}^{n} \delta_{x} c_{i+1 / 2}^{\widehat{n}}
$$

The computational procedure of the uncoupled leap-frog scheme at each time step is listed below:

Step 1. The vapor concentration $c_{j}^{n+1}$ can be calculated by solving the tridiagonal linear systems defined in (18)-(20).

Step 2. With the updated vapor concentration $c_{j}^{n+1}$, we can get $c_{0}^{\widehat{n}}$ and $u_{i+1 / 2}^{\widehat{n}}$ correspondingly.

Step 3. Finally, the temperature $\theta_{j}^{n+1}$ can be obtained by solving the tridiagonal linear system (21)-(23).

\section{The Leap-Frog Scheme and the Optimal Error Estimate}

In this section, we will show the existence and uniqueness of the solution to the system (18)-(26) with optimal error estimates in the discrete $L^{2}$ norm. Let $v=\left\{v_{j}^{n}\right\}_{j=0}^{M}$ and $z=$ $\left\{z_{j}\right\}_{j=0}^{M}$ be two mesh functions on $\Omega_{h}$. We define the inner product and norms by

$$
\begin{aligned}
(v, z) & =h\left(\frac{1}{2} v_{0} z_{0}+\sum_{i=1}^{M-1} v_{i} z_{i}+\frac{1}{2} v_{M} z_{M}\right) \\
\|v\| & =\sqrt{h\left[\frac{1}{2}\left(v_{0}\right)^{2}+\sum_{i=1}^{M-1}\left(v_{i}\right)^{2}+\frac{1}{2}\left(v_{M}\right)^{2}\right]} \\
\|v\|_{\infty} & =\max _{0 \leq i \leq M}\left|v_{i}\right|, \\
\left\|\delta_{x} v\right\| & =\sqrt{h \sum_{i=0}^{M-1}\left(\frac{v_{i+1}-v_{i}}{h}\right)^{2}} \\
\left\|\delta_{x}^{2} v\right\| & =\sqrt{h \sum_{i=1}^{M-1}\left(\delta_{x}^{2} v_{i}\right)^{2}} .
\end{aligned}
$$

Let $(C, \Theta)$ be the solution of the system (6)-(12) and $C_{i}^{n}=$ $c\left(x_{i}, t_{n}\right), \Theta_{i}^{n}=\theta\left(x_{i}, t_{n}\right)$. The error functions are defined by

$$
\begin{aligned}
& \widetilde{c}_{i}^{n}=C_{i}^{n}-c_{i}^{n}, \\
& \widetilde{\theta}_{i}^{n}=\Theta_{i}^{n}-\theta_{i}^{n},
\end{aligned}
$$

$$
0 \leq i \leq M, 0 \leq n \leq N .
$$

We state our main result in the theorem below:

Theorem 1. Suppose that the solution $(c, v)$ of the system (6)(12) is in $C^{4,3}([0, L] \times[0, \mathscr{T}])$, satisfying (13). Then there exist positive constants $h_{0}$ and $E_{0}$, independent of $h$ and $\tau$, such that, 
when $\tau \leq E_{c} h \leq h_{0}$, the finite difference scheme (18)-(26) is uniquely solvable and

$$
\begin{aligned}
& \left\|\widetilde{c}^{n+1}\right\|^{2}+\left\|\widetilde{\theta}^{n+1}\right\|^{2}+\tau \sum_{m=1}^{n}\left(\left\|\delta_{x} \widetilde{c}^{\widehat{n}}\right\|^{2}+\left\|\delta_{x} \widetilde{\theta}^{\widehat{n}}\right\|^{2}\right) \\
& \leq E_{0}\left(\tau^{2}+h^{2}\right)^{2}, \quad 1 \leq n \leq N-1 .
\end{aligned}
$$

To prove the theorem, we make a stronger assumption that there exists $E_{0}>0$, independent of $n, h, \tau$, such that the inequality,

$$
\left\|\widetilde{c}^{n+1}\right\|^{2}+\left\|\widetilde{\theta}^{n+1}\right\|^{2}+\left\|\delta_{x} \widetilde{\theta}^{n+1}\right\|^{2} \leq E_{0}\left(\tau^{2}+h^{2}\right)^{2},
$$

holds for $n \leq k-1$. We prove the assumption and the theorem by induction method. By the initial condition (26), this is true for $n=0$. In the next subsection we will show that this is also true for $n=1$. In this part, we let $E_{c}$ be a generic positive constant, which is associated with the physical parameters $\sigma$, $\lambda, \kappa, c_{\min }, c_{\max }, \theta_{\min }, \theta_{\max }$, the parameters involved in initial and boundary conditions and the solution of the system (6)(12). $E_{c}$ is independent of time step $n$, mesh size $h, \tau$, and constant $E_{0}$.

3.1. The Leap-Frog Scheme and Preliminaries. For convenience of calculations, we further introduce some notations. Let $u=(c \theta)_{x}, w=\theta_{x}$; thus the sweat transport system (6)-(7) can be reduced to

$$
\begin{aligned}
& c_{t}-u_{x}=-\Gamma(c, \theta), \\
& 0 \leq x \leq L, 0<t \leq \mathscr{T}, \\
& u=\left(c \theta_{x}+\theta c_{x}\right), \\
& 0 \leq x \leq L, 0<t \leq \mathscr{T}, \\
& 0 \leq x \leq L, 0<t \leq \mathscr{T}, \\
& 0 \leq x \leq L, 0<t \leq \mathscr{T},
\end{aligned}
$$

with the initial and boundary conditions

$$
\begin{aligned}
u=\alpha_{1}\left(c-\mu_{1}\right), & x=0,0<t \leq \mathscr{T}, \\
u=\alpha_{2}\left(\mu_{2}-c\right), & x=L, 0<t \leq \mathscr{T}, \\
w & =\beta_{1}\left(\theta-v_{1}\right), \quad x=0,0<t \leq \mathscr{T}, \\
w & =\beta_{2}\left(v_{2}-\theta\right), \quad x=L, 0<t \leq \mathscr{T}, \\
c(x, 0) & =c_{0}(x), \\
\theta(x, 0) & =\theta_{0}(x),
\end{aligned}
$$

$0 \leq x \leq L$
The discrete leap-frog system (18)-(23) is modified as

$$
\begin{aligned}
& \nabla_{t} c_{i}^{n}-\delta_{x}^{*} u_{i}^{\widehat{n}}=-\Gamma\left(c_{i}^{n}, \theta_{i}^{n}\right), \quad 1 \leq i \leq M-1, \\
& \nabla_{t} c_{0}^{n}-\frac{2}{h}\left[u_{1 / 2}^{\widehat{n}}-\alpha_{1}\left(c_{0}^{\widehat{n}}-\mu_{1}\right)\right]=-\Gamma\left(c_{0}^{n}, \theta_{0}^{n}\right), \\
& \nabla_{t} c_{M}^{n}-\frac{2}{h}\left[\alpha_{2}\left(\mu_{2}-c_{M}^{\widehat{n}}\right)-u_{M-1 / 2}^{\widehat{n}}\right]=-\Gamma\left(c_{M}^{n}, \theta_{M}^{n}\right), \\
& u_{i+1 / 2}^{\widehat{n}}=c_{i+1 / 2}^{n} \delta_{x} \theta_{i+1 / 2}^{n}+\theta_{i+1 / 2}^{n} \delta_{x} c_{i+1 / 2}^{\widehat{n}}, \\
& \left(c_{i}^{\widehat{n}}+\sigma\right) \nabla_{t} \theta_{i}^{n}-\frac{1}{2}\left(u_{i-1 / 2}^{\widehat{n}} \delta_{x} \theta_{i-1 / 2}^{\widehat{n}}+u_{i+1 / 2}^{\widehat{n}} \delta_{x} \theta_{i+1 / 2}^{\widehat{n}}\right) \\
& \quad-\kappa \delta_{x}^{*} w_{i}^{\widehat{n}}=\left(\lambda+\theta_{i}^{n}\right) \Gamma\left(c_{i}^{n}, \theta_{i}^{n}\right), \quad 1 \leq i \leq M-1, \\
& \left(c_{0}^{\widehat{n}}+\sigma\right) \nabla_{t} \theta_{0}^{n}-u_{1 / 2}^{\widehat{n}} \delta_{x} \theta_{1 / 2}^{\widehat{n}} \\
& \quad-\frac{2 \kappa}{h}\left[w_{1 / 2}^{\widehat{n}}-\beta_{1}\left(\theta_{0}^{\widehat{n}}-v_{1}\right)\right]=\left(\lambda+\theta_{0}^{n}\right) \Gamma\left(c_{0}^{n}, \theta_{0}^{n}\right), \\
& \left(c_{M}^{\widehat{n}}+\sigma\right) \nabla_{t} \theta_{M}^{n}-u_{M-1 / 2}^{\widehat{n}} \delta_{x} \theta_{M-1 / 2}^{\widehat{n}} \\
& \quad-\frac{2 \kappa}{h}\left[\beta_{2}\left(v_{2}-\theta_{M}^{\widehat{n}}\right)-w_{M-1 / 2}^{\widehat{n}}\right] \\
& \quad=\left(\lambda+\theta_{M}^{n}\right) \Gamma\left(c_{M}^{n}, \theta_{M}^{n}\right), \\
& w_{i+1 / 2}^{\hat{n}}=\delta_{x} \theta_{i+1 / 2}^{\widehat{n}}, \quad 0 \leq i \leq M-1, \quad 1 \leq n \leq N-1 .
\end{aligned}
$$

Let $U_{i}^{n}=u\left(x_{i}, t_{n}\right)=(c \theta)_{x}\left(x_{i}, t_{n}\right)$ and $W_{i}^{n}=w\left(x_{i}, t_{n}\right)=$ $\theta_{x}\left(x_{i}, t_{n}\right)$. We denote by $u_{i}^{n}$ and $w_{i}^{n}$ the corresponding finite difference solution and

$$
\begin{gathered}
\widetilde{u}_{i+1 / 2}^{n}=U_{i+1 / 2}^{n}-u_{i+1 / 2}^{n}, \\
\widetilde{w}_{i+1 / 2}^{n}=W_{i+1 / 2}^{n}-w_{i+1 / 2}^{n},
\end{gathered}
$$

$$
0 \leq i \leq M-1,1 \leq n \leq N-1 .
$$

We get

$$
\begin{aligned}
& \nabla_{t} C_{i}^{n}-\delta_{x}^{*} U_{i}^{\widehat{n}}=-\Gamma\left(C_{i}^{n}, \Theta_{i}^{n}\right)+R_{c, i}^{n}, \quad 1 \leq i \leq M-1, \\
& \nabla_{t} C_{0}^{n}-\frac{2}{h}\left[U_{1 / 2}^{\widehat{n}}-\alpha_{1}\left(C_{0}^{\widehat{n}}-\mu_{1}\right)\right]=-\Gamma\left(C_{0}^{n}, \Theta_{0}^{n}\right)+R_{c, 0}^{n} \\
& \nabla_{t} C_{M}^{n}-\frac{2}{h}\left[\alpha_{2}\left(\mu_{2}-C_{M}^{\widehat{n}}\right)-U_{M-1 / 2}^{\widehat{n}}\right]=-\Gamma\left(C_{M}^{n}, \Theta_{M}^{n}\right) \\
& \quad+R_{c, M}^{n}, \\
& U_{i+1 / 2}^{\widehat{n}}=C_{i+1 / 2}^{n} \delta_{x} \Theta_{i+1 / 2}^{n}+\Theta_{i+1 / 2}^{n} \delta_{x} C_{i+1 / 2}^{\widehat{n}}+R_{u, i+1 / 2}^{n}, \\
& \quad 0 \leq i \leq M-1, \\
& \left(C_{i}^{\widehat{n}}+\sigma\right) \nabla_{t} \Theta_{i}^{n}-\frac{1}{2}\left(U_{i-1 / 2}^{\widehat{n}} \delta_{x} \Theta_{i-1 / 2}^{\widehat{n}}+U_{i+1 / 2}^{\widehat{n}} \delta_{x} \Theta_{i+1 / 2}^{\widehat{n}}\right) \\
& \quad-\kappa \delta_{x}^{*} W_{i}^{\widehat{n}}=\left(\lambda+\Theta_{i}^{n}\right) \Gamma\left(C_{i}^{n}, \Theta_{i}^{n}\right)+R_{\theta, i}^{n},
\end{aligned}
$$




$$
\begin{aligned}
& \left(C_{0}^{\widehat{\hat{n}}}+\sigma\right) \nabla_{t} \Theta_{0}^{n}-U_{1 / 2}^{n} \delta_{x} \Theta_{1 / 2}^{\widehat{\hat{n}}} \\
& -\frac{2 \kappa}{h}\left[W_{1 / 2}^{\widehat{n}}-\beta_{1}\left(\Theta_{0}^{\widehat{n}}-v_{1}\right)\right] \\
& =\left(\lambda+\Theta_{0}^{n}\right) \Gamma\left(C_{0}^{n}, \Theta_{0}^{n}\right)+R_{\theta, 0}^{n}, \\
& \left(C_{M}^{\widehat{n}}+\sigma\right) \nabla_{t} \Theta_{M}^{n}-U_{M-1 / 2}^{\widehat{n}} \delta_{x} \Theta_{M-1 / 2}^{\widehat{n}} \\
& -\frac{2 \kappa}{h}\left[\beta_{2}\left(v_{2}-\Theta_{M}^{\widehat{n}}\right)-W_{M-1 / 2}^{\widehat{n}}\right] \\
& =\left(\lambda+\Theta_{M}^{n}\right) \Gamma\left(C_{M}^{n}, \Theta_{M}^{n}\right)+R_{\theta, M}^{n}, \\
& W_{i+1 / 2}^{\widehat{n}}=\delta_{x} \Theta_{i+1 / 2}^{\widehat{n}}+R_{w, i+1 / 2}^{n}, \\
& \quad 0 \leq i \leq M-1, \quad 1 \leq n \leq N-1,
\end{aligned}
$$

and the initial conditions

$$
\begin{aligned}
& C_{i}^{0}=c_{0}\left(x_{i}\right), \\
& \Theta_{i}^{0}=\theta_{0}\left(x_{i}\right), \\
& C_{i}^{1}=c_{0}\left(x_{i}\right)+\tau c_{t}\left(x_{i}, 0\right)+\tau R_{c, i}^{1}, \\
& \Theta_{i}^{1}=\theta_{0}\left(x_{i}\right)+\tau \theta_{t}\left(x_{i}, 0\right)+\tau R_{\theta, i}^{1}
\end{aligned}
$$

where

$$
\begin{aligned}
\left|R_{\theta, i}^{1}\right|,\left|R_{\theta, i}^{1}\right| \leq E_{1}\left(\tau+h^{2}\right), & 0 \leq i \leq M . \\
\left|R_{c, i}^{n}\right|,\left|R_{\theta, i}^{n}\right| \leq E_{2}\left(\tau^{2}+h^{2}\right), & 1 \leq i \leq M-1, \\
\left|R_{u, i+1 / 2}^{n}\right|,\left|R_{w, i+1 / 2}^{n}\right| \leq E_{2}\left(\tau^{2}+h^{2}\right), & 0 \leq i \leq M-1, \\
\left|R_{c, 0}^{n}\right|,\left|R_{c, M}^{n}\right| \leq E_{2}\left(\tau^{2}+h^{2}\right), & \\
\left|R_{\theta, 0}^{n}\right|,\left|R_{\theta, M}^{n}\right| \leq E_{2}\left(\tau^{2}+h\right), & 1 \leq n \leq N-1 .
\end{aligned}
$$

Subtracting the system (36) from the system (38), we get the error equations

$$
\begin{aligned}
& \nabla_{t} \widetilde{c}_{i}^{n}-\delta_{x}^{*} \widetilde{u}_{i}^{\widehat{n}}=-\left[\Gamma\left(C_{i}^{n}, \Theta_{i}^{n}\right)-\Gamma\left(c_{i}^{n}, \theta_{i}^{n}\right)\right]+R_{c, i}^{n} \\
& \quad:=\bar{R}_{c, i}^{n}, \quad 1 \leq i \leq M-1, \\
& \nabla_{t} \widetilde{c}_{0}^{n}-\frac{2}{h}\left[\widetilde{u}_{1 / 2}^{\widehat{n}}-\alpha_{1} \widehat{c}_{0}^{\hat{n}}\right]=-\left[\Gamma\left(C_{0}^{n}, \Theta_{0}^{n}\right)-\Gamma\left(c_{0}^{n}, \theta_{0}^{n}\right)\right] \\
& \quad+R_{c, 0}^{n}:=\bar{R}_{c, 0}^{n}, \\
& \nabla_{t} \widetilde{c}_{M}^{n}-\frac{2}{h}\left[-\alpha_{2} \widehat{c}_{M}^{\widehat{n}}-\widetilde{u}_{M-1 / 2}^{\widehat{n}}\right] \\
& \quad=-\left[\Gamma\left(C_{M}^{n}, \Theta_{M}^{n}\right)-\Gamma\left(c_{M}^{n}, \theta_{M}^{n}\right)\right]+R_{c, M}^{n}:=\bar{R}_{c, M}^{n}, \\
& \widetilde{u}_{i+1 / 2}^{\widehat{n}}=\widetilde{c}_{i+1 / 2}^{n} \delta_{x} \Theta_{i+1 / 2}^{n}+\widetilde{\theta}_{i+1 / 2}^{n} \delta_{x} C_{i+1 / 2}^{\widehat{n}} \\
& \quad+c_{i+1 / 2}^{n} \delta_{x} \widetilde{\theta}_{i+1 / 2}^{n}+\theta_{i+1 / 2}^{n} \delta_{x} \widehat{c}_{i+1 / 2}^{\hat{n}}+R_{u, i+1 / 2}^{n}, \\
& \quad 0 \leq i \leq M-1,
\end{aligned}
$$

$$
\begin{aligned}
& \left(c_{i}^{\widehat{n}}+\sigma\right) \nabla_{t} \widetilde{\theta}_{i}^{n}-\frac{1}{2}\left(u_{i-1 / 2}^{\widehat{n}} \delta_{x} \widetilde{\theta}_{i-1 / 2}^{\widehat{n}}+u_{i+1 / 2}^{\widehat{n}} \delta_{x} \widetilde{\theta}_{i+1 / 2}^{\hat{n}}\right) \\
& -\kappa \delta_{x}^{*} \widetilde{w}_{i}^{\widehat{n}}=-\widehat{c}_{i}^{\widehat{n}} \nabla_{t} \Theta_{i}^{n} \\
& +\frac{1}{2}\left(\widetilde{u}_{i-1 / 2}^{\widehat{n}} \delta_{x} \Theta_{i-1 / 2}^{\widehat{n}}+\widetilde{u}_{i+1 / 2}^{\widehat{n}} \delta_{x} \Theta_{i+1 / 2}^{\widehat{n}}\right) \\
& +\left[\left(\lambda+\Theta_{i}^{n}\right) \Gamma\left(C_{i}^{n}, \Theta_{i}^{n}\right)-\left(\lambda+\theta_{i}^{n}\right) \Gamma\left(c_{i}^{n}, \theta_{i}^{n}\right)\right] \\
& +R_{\theta, i}^{n}:=\bar{R}_{\theta, i}^{n}, \quad 1 \leq i \leq M-1, \\
& \left(c_{0}^{\widehat{n}}+\sigma\right) \nabla_{t} \widetilde{\theta}_{0}^{n}-u_{1 / 2}^{\widehat{n}} \delta_{x} \widetilde{\theta}_{1 / 2}^{\widehat{n}}-\frac{2 \kappa}{h}\left[\widetilde{w}_{1 / 2}^{\widehat{n}}-\beta_{1} \widetilde{\theta}_{0}^{\widehat{n}}\right] \\
& =-\widehat{c}_{0}^{\widehat{n}} \nabla_{t} \Theta_{0}^{\widehat{n}}+\widetilde{u}_{1 / 2}^{\widehat{n}} \delta_{x} \Theta_{1 / 2}^{\widehat{n}} \\
& +\left[\left(\lambda+\Theta_{0}^{n}\right) \Gamma\left(C_{0}^{n}, \Theta_{0}^{n}\right)-\left(\lambda+\theta_{0}^{n}\right) \Gamma\left(c_{0}^{n}, \theta_{0}^{n}\right)\right] \\
& +R_{\theta, 0}^{n}:=\bar{R}_{\theta, 0}^{n} \\
& \left(c_{M}^{\widehat{n}}+\sigma\right) \nabla_{t} \widetilde{\theta}_{M}^{n}-u_{M-1 / 2}^{\widehat{n}} \delta_{x} \widetilde{\theta}_{M-1 / 2}^{\widehat{n}} \\
& -\frac{2 \kappa}{h}\left[-\beta_{2} \widetilde{\theta}_{M}^{\hat{n}}-\widetilde{w}_{M-1 / 2}^{\widehat{n}}\right]=-\widehat{c}_{M}^{\widehat{n}} \nabla_{t} \Theta_{M}^{n} \\
& +\widetilde{u}_{M-1 / 2}^{\widehat{n}} \delta_{x} \Theta_{M-1 / 2}^{\widehat{n}} \\
& +\left[\left(\lambda+\Theta_{M}^{n}\right) \Gamma\left(C_{M}^{n}, \Theta_{M}^{n}\right)-\left(\lambda+\theta_{M}^{n}\right) \Gamma\left(c_{M}^{n}, \theta_{M}^{n}\right)\right] \\
& +R_{\theta, M}^{n}:=\bar{R}_{\theta, M}^{n}, \\
& \widetilde{w}_{i+1 / 2}^{\widehat{n}}=\delta_{x} \widetilde{\theta}_{i+1 / 2}^{\widehat{n}}+R_{w, i+1 / 2}^{n}, \\
& 0 \leq i \leq M-1,1 \leq n \leq N-1,
\end{aligned}
$$

and

$$
\begin{aligned}
\widetilde{c}_{i}^{0} & =0, \\
\widetilde{\theta}_{i}^{0} & =0, \\
\widetilde{c}_{i}^{1} & =\tau R_{c, i}^{1}, \\
\widetilde{\theta}_{i}^{1} & =\tau R_{\theta, i}^{1},
\end{aligned}
$$

and by (40), we can directly derive the inequality

$$
\left\|\widetilde{c}^{1}\right\|^{2}+\left\|\widetilde{\theta}^{1}\right\|^{2}+\left\|\delta_{x} \widetilde{\theta}^{1}\right\|^{2} \leq E_{0}\left(\tau^{2}+h^{2}\right)^{2} .
$$

To prove our main theorem, the following formula will be often used:

$$
\begin{aligned}
& {\left[v_{1 / 2} z_{0}+h \sum_{i=1}^{M-1} \delta_{x} v_{i} z_{i}-v_{M-1 / 2} z_{M}\right]} \\
& =\sum_{i=0}^{M-1} v_{i+1 / 2}\left(z_{i}^{k}-z_{i+1}^{k}\right) .
\end{aligned}
$$

In the following lemma, we present discrete Sobolev interpolation formulas and the proof can be found in [24]. 
Lemma 2. Let $v$ and $z$ be two mesh functions. Then for any positive constant $\epsilon$,

$$
\begin{gathered}
\|v\|_{\infty}^{2} \leq \epsilon\left\|\delta_{x} v\right\|^{2}+\left(\frac{1}{\epsilon}+\frac{1}{L}\right)\|v\|^{2}, \\
\left\|\delta_{x} v\right\|_{\infty} \leq \epsilon\left\|\delta_{x}^{2} v\right\|+E_{c}\left\|\delta_{x} v\right\|^{2} .
\end{gathered}
$$

Lemma 3.

$$
\begin{aligned}
\left\|\widetilde{c}^{n}\right\|_{\infty},\left\|\widetilde{\theta}^{n}\right\|_{\infty} & \leq 3 E_{0}^{1 / 2}\left(\tau^{7 / 4}+h^{3 / 2}\right), \quad 1 \leq n \leq k, \\
\left\|\widetilde{c}_{x}\right\|,\left\|\widetilde{\theta}_{x}^{\hat{n}}\right\| & \leq E_{0}^{1 / 2}\left(\tau^{3 / 2}+h\right), \quad 1 \leq n \leq k-1 .
\end{aligned}
$$

Proof. From (30) for $0 \leq n \leq k-1$, we have

$$
\begin{gathered}
\left\|\widetilde{c}^{n+1}\right\|^{2}+\tau\left\|\delta_{x} \widetilde{c}^{n+1}\right\|^{2} \leq E_{0}\left(\tau^{2}+h^{2}\right)^{2}, \\
\left\|\widetilde{\theta}^{n+1}\right\|^{2}+\tau\left\|\delta_{x} \widetilde{\theta}^{n+1}\right\|^{2} \leq E_{0}\left(\tau^{2}+h^{2}\right)^{2} .
\end{gathered}
$$

When $\tau \leq h$, with the inverse inequality we have

$$
\begin{aligned}
\left|\vec{c}_{i}^{n+1}\right|^{2} & \leq\left\|\widetilde{c}^{n+1}\right\|_{\infty}^{2} \leq 2 h^{-1}\left\|\widetilde{c}^{n+1}\right\|^{2} \leq 2 h^{-1} E_{0}\left(\tau^{2}+h^{2}\right)^{2} \\
& \leq 8 E_{0} h^{3} .
\end{aligned}
$$

When $h \leq \tau$, by taking $\epsilon=\tau^{1 / 2}$ in Lemma 2,

$$
\begin{aligned}
\left|\vec{c}_{i}^{n+1}\right|^{2} & \leq\left\|\vec{c}^{n+1}\right\|_{\infty}^{2} \\
& \leq \tau^{1 / 2}\left\|\delta_{x} \vec{c}^{n+1}\right\|^{2}+\left(\tau^{-1 / 2}+L^{-1}\right)\left\|\vec{c}^{n+1}\right\|^{2} \\
& \leq\left(\tau^{-1 / 2}+\tau^{1 / 2} L^{-1}\right) E_{0}\left(\tau^{2}+h^{2}\right)^{2} \leq 8 E_{0} \tau^{7 / 2}, \\
& 1 \leq i \leq M-1,0 \leq n \leq k-1 .
\end{aligned}
$$

The first part of (54) is obtained and the second part and the inequality (55) can be proved similarly.

In addition, by Lemma 3, there exist constants $E_{3}>0$ and $s_{0}>0$ such that, when $h, \tau \leq s_{0}$,

$$
\begin{aligned}
& \frac{c_{\min }}{2} \leq c_{i}^{n} \leq 2 c_{\max }, \\
& \frac{\theta_{\min }}{2} \leq \theta_{i}^{n} \leq 2 \theta_{\max },
\end{aligned}
$$

$$
0 \leq i \leq M-1,1 \leq n \leq k,
$$

and

$$
\begin{aligned}
& \left|\nabla_{t} \Theta_{i}^{n}\right|,\left|\nabla_{t} C_{i}^{n}\right| \leq E_{3}, \quad 0 \leq i \leq M-1, \quad 1 \leq n \leq k, \\
& \left|\delta_{x} C_{i+1 / 2}^{n}\right|,\left|\delta_{x} \Theta_{i+1 / 2}^{n}\right|,\left|\delta_{x} C_{i+1 / 2}^{\widehat{n}}\right|,\left|\delta_{x} \Theta_{i+1 / 2}^{\widehat{n}}\right| \leq E_{3}, \\
& 0 \leq i \leq M-1,1 \leq n \leq k \text {, } \\
& \left|c_{i+1 / 2}^{n}\right|,\left|\theta_{i+1 / 2}^{n}\right|, \leq E_{3}, \quad 0 \leq i \leq M-1,1 \leq n \leq k,
\end{aligned}
$$

$$
\begin{aligned}
& \left|\Gamma\left(c_{i+1 / 2}^{n}, \theta_{i+1 / 2}^{n}\right)\right| \leq E_{3}, \quad 0 \leq i \leq M-1,1 \leq n \leq k, \\
& \left|\left(\lambda+\Theta_{i}^{n}\right) \Gamma\left(C_{i}^{n}, \Theta_{i}^{n}\right)-\left(\lambda+\theta_{i}^{n}\right) \Gamma\left(c_{i}^{n}, \theta_{i}^{n}\right)\right| \\
& \quad \leq E_{3}\left(\left|\widetilde{c}_{i}^{n}\right|+\left|\widetilde{\theta}_{i}^{n}\right|\right), \quad 0 \leq i \leq M, \quad 1 \leq n \leq k, \\
& \left|\Gamma\left(C_{i}^{n}, \Theta_{i}^{n}\right)-\Gamma\left(c_{i}^{n}, \theta_{i}^{n}\right)\right| \leq E_{3}\left(\left|\widetilde{c}_{i}^{n}\right|+\left|\widetilde{\theta}_{i}^{n}\right|\right), \\
& 0 \leq i \leq M, 1 \leq n \leq k .
\end{aligned}
$$

3.2. The Existence and Uniqueness. Since the coefficient matrix in the system (18)-(20) is strictly diagonally dominant, thus the system (18)-(20) has a unique solution $c_{i}^{k+1}$. Here we will discuss the boundedness of $c_{i}^{k+1}$.

Multiplying (41)-(43) by $h \widetilde{c}_{i}^{\hat{n}}, h \widehat{c}_{0}^{\hat{n}} / 2$, and $h \widehat{c}_{M}^{\hat{n}} / 2$, respectively, we get

$$
\begin{aligned}
& \left(\nabla_{t} \widetilde{c}^{n}, \widehat{c}^{\hat{n}}\right)+\alpha_{1}\left|\widehat{c}_{0}^{\hat{n}}\right|^{2}+\alpha_{2}\left|\widehat{c}_{M}^{\hat{n}}\right|^{2}=\left[\widetilde{u}_{1 / 2}^{\hat{n}} \widehat{c}_{0}^{\widehat{n}}\right. \\
& \left.+h \sum_{i=1}^{M-1} \delta_{x}^{*} \widetilde{u}_{i}^{\widehat{n}} \widehat{c}_{i}^{\widehat{n}}+\widetilde{u}_{M-1 / 2}^{\widehat{n}} \widehat{c}_{M}^{\widehat{n}}\right]+h \sum_{i=1}^{M-1} R_{c, i}^{n} \widehat{c}_{i}^{\widehat{n}}+\frac{h}{2} \\
& \cdot R_{c, 0}^{n} \widehat{c}_{0}^{\hat{n}}+\frac{h}{2} R_{c, M}^{n} \widehat{c}_{M}^{\widehat{n}} \\
& +h\left\{\frac{1}{2}\left[\Gamma\left(C_{0}^{n}, \Theta_{0}^{n}\right)-\Gamma\left(c_{0}^{n}, \theta_{0}^{n}\right)\right] \widehat{c}_{0}^{\widehat{n}}\right. \\
& +\sum_{i=1}^{M-1}\left[\Gamma\left(C_{i}^{n}, \Theta_{i}^{\mathrm{n}}\right)-\Gamma\left(c_{i}^{n}, \theta_{i}^{n}\right)\right] \widehat{c}_{i}^{\widehat{n}} \\
& \left.+\frac{1}{2}\left[\Gamma\left(C_{M}^{n}, \Theta_{M}^{n}\right)-\Gamma\left(c_{M}^{n}, \theta_{M}^{n}\right)\right] \widehat{c}_{M}^{\hat{n}}\right\}:=J_{1}+J_{2} \\
& +J_{3}, \quad 1 \leq n \leq k .
\end{aligned}
$$

With (44), (51), (59), (60), and (40), we see that

$$
\begin{aligned}
& -J_{1}=-\widetilde{u}_{1 / 2}^{\widehat{n}} \widetilde{c}_{0}^{\widehat{n}}-h \sum_{i=1}^{M-1} \delta_{x} \widetilde{u}_{i}^{\widehat{n}} \widetilde{c}_{i}^{\widehat{n}}+\widetilde{u}_{\mathrm{M}-1 / 2}^{\widehat{n}} \widetilde{c}_{M}^{\widehat{n}} \\
& =h \sum_{i=0}^{M-1} \widetilde{u}_{i+1 / 2}^{\widehat{n}} \delta_{x} \widetilde{c}_{i+1 / 2}^{\widehat{n}}=h \sum_{i=0}^{M-1}\left[\widetilde{c}_{i+1 / 2}^{n} \delta_{x} \Theta_{i+1 / 2}^{n}\right. \\
& +\widetilde{\theta}_{i+1 / 2}^{n} \delta_{x} C_{i+1 / 2}^{\widehat{n}}+c_{i+1 / 2}^{n} \delta_{x} \widetilde{\theta}_{i+1 / 2}^{n}+\theta_{i+1 / 2}^{n} \delta_{x} \widehat{c}_{i+1 / 2}^{\hat{n}} \\
& \left.+R_{u, i+1 / 2}^{n}\right] \delta_{x} \widetilde{c}_{i+1 / 2}^{\widehat{n}} \geq \frac{\theta_{\min }}{2}\left\|\delta_{x} \widehat{c}^{\hat{n}}\right\|^{2}-2 c_{\max }\left\|\delta_{x} \widetilde{\theta}^{n}\right\| \\
& \cdot\left\|\delta_{x} \widetilde{c}^{\hat{n}}\right\|-E_{3}\left(\left\|\widetilde{c}^{n}\right\|+\left\|\widetilde{\theta}^{n}\right\|\right)\left\|\delta_{x} \widehat{c}^{\hat{n}}\right\| \\
& -h \sum_{i=0}^{M-1}\left|R_{u, i+1 / 2}^{n}\right|\left|\delta_{x} \widetilde{c}_{i+1 / 2}\right| \geq \frac{\theta_{\min }}{4}\left\|\delta_{x} \widetilde{c}^{\hat{n}}\right\|^{2} \\
& -E_{c}\left(\left\|\delta_{x} \widetilde{\theta}^{n}\right\|^{2}+\left\|\widetilde{c}^{n}\right\|^{2}+\left\|\widetilde{\theta}^{n}\right\|^{2}\right)-E_{c}\left(\tau^{2}+h^{2}\right)^{2},
\end{aligned}
$$


and by using (60) again, we have

$$
\left|J_{3}\right| \leq E_{3}\left(\left\|\widetilde{c}^{n}\right\|+\left\|\widetilde{\theta}^{n}\right\|\right)\left\|\vec{c}^{\hat{n}}\right\|,
$$

and with (52),

$$
\begin{aligned}
\left|J_{2}\right| \leq & \frac{1}{2} h \sum_{i=1}^{M-1}\left[\left(R_{c, i}^{n}\right)^{2}+\left(\widehat{c}_{i}^{\hat{n}}\right)^{2}\right]+\frac{h^{2}}{2}\left|R_{c, 0}^{n}\right|^{2}+\frac{1}{2}\left|\widehat{c}_{0}\right|^{2} \\
& +\frac{h^{2}}{2}\left|R_{c, M}^{n}\right|^{2}+\frac{1}{2}\left|\widehat{c}_{M}^{\hat{n}}\right|^{2} \\
\leq & \left\|\widehat{c^{2}}\right\|_{\infty}^{2}+E_{c}\left\|\widehat{c^{n}}\right\|^{2}+\frac{E_{2} L}{2}\left(\tau^{2}+h^{2}\right)^{2} \\
& +E_{2} h^{2}\left(\tau^{2}+h^{2}\right)^{2} \\
\leq & \frac{\theta_{\min }}{8}\left\|\delta_{x} \widehat{c^{n}}\right\|^{2}+E_{c}\left\|\vec{c}^{\hat{n}}\right\|^{2}+E_{c}\left(\tau^{2}+h^{2}\right)^{2} .
\end{aligned}
$$

Substituting the last three equations into (61) results in

$$
\begin{gathered}
\frac{1}{2} \nabla_{t}\left\|\widetilde{c}^{n}\right\|^{2}+\frac{\theta_{\min }}{8}\left\|\delta_{x} \widetilde{c}^{\hat{c}}\right\|^{2}+\alpha_{1}\left|\widetilde{c}_{0}^{n}\right|^{2}+\alpha_{2}\left|\widehat{c}_{M}^{\hat{n}}\right|^{2} \\
\leq E_{c}\left(\left\|\delta_{x} \widetilde{\theta}^{n}\right\|^{2}+\left\|\widetilde{c}^{n}\right\|^{2}+\left\|\widetilde{\theta}^{n}\right\|^{2}+\left\|\widetilde{c}^{\hat{n}}\right\|^{2}\right) \\
+E_{c}\left(\tau^{2}+h^{2}\right)^{2}, \quad 1 \leq n \leq k,
\end{gathered}
$$

where we have noted $\left(\nabla_{t} \widetilde{c}^{n}, \widehat{c}^{n}\right)=(1 / 4 \tau)\left(\left\|\widetilde{c}^{n+1}\right\|^{2}-\left\|\tilde{c}^{n-1}\right\|^{2}\right)=$ $(1 / 2) \nabla_{t}\left\|\tilde{c}^{n}\right\|^{2}$. Moreover, by the assumption of the induction,

$$
\begin{aligned}
& \left\|\widetilde{c}^{k+1}\right\|^{2}+4 \tau\left(\frac{\theta_{\min }}{8}\left\|\delta_{x} \bar{c}^{\hat{k}}\right\|^{2}+\alpha_{1}\left|\widehat{c}_{0}^{\hat{k}}\right|^{2}+\alpha_{2}\left|\widehat{c}_{M}^{\hat{k}}\right|^{2}\right) \\
& \leq\left\|\widehat{c}^{k-1}\right\|^{2} \\
& \quad+4 E_{c} \tau\left(\left\|\delta_{x} \widetilde{\theta}^{k}\right\|^{2}+\left\|\widetilde{c}^{k}\right\|^{2}+\left\|\widetilde{\theta}^{k}\right\|^{2}+\left\|\hat{c}^{\hat{k}}\right\|^{2}\right) \\
& \quad+4 \tau E_{c}\left(\tau^{2}+h^{2}\right)^{2} .
\end{aligned}
$$

Since we have the fact that $\left\|\widehat{c}^{\widehat{k}}\right\|^{2} \leq(1 / 2)\left(\left\|\bar{c}^{k+1}\right\|^{2}+\left\|\widetilde{c}^{k-1}\right\|^{2}\right)$, thus

$$
\begin{aligned}
& \left(1-2 \tau E_{c}\right)\left\|\vec{c}^{k+1}\right\|^{2}+\frac{\tau \theta_{\min }}{2}\left\|\delta_{x} \widehat{c}^{\hat{c}}\right\|^{2} \\
& \quad \leq\left(E_{0}+4 \tau E_{c}+4 E_{c} E_{0}+6 \tau E_{c} E_{0}\right)\left(\tau^{2}+h^{2}\right)^{2} .
\end{aligned}
$$

When $\tau E_{c}<1 / 4$, we can get the inequality as

$$
\left\|\widehat{c}^{k+1}\right\|^{2}+\tau \theta_{\min }\left\|\delta_{x} \bar{c}^{\hat{c}}\right\|^{2} \leq E_{4}\left(\tau^{2}+h^{2}\right)^{2} .
$$

Since $E_{4}$ are independent of $k$, by (13) when $h$ and $\tau$ are small enough,

$$
c_{i}^{k+1} \geq 0, \quad 0 \leq i \leq M .
$$

Now we try to prove our main theorem. By noting (44), (60), (40), and Lemma 3,

$$
\begin{aligned}
& \left\|\widetilde{u}^{\hat{n}}\right\|^{2}=\sum_{i=0}^{M-1} h\left[c_{i+1 / 2}^{n} \delta_{x} \widetilde{\theta}_{i+1 / 2}^{n}+\theta_{i+1 / 2}^{n} \delta_{x} \widetilde{c}_{i+1 / 2}^{\widehat{n}}\right. \\
& \left.+\widetilde{c}_{i+1 / 2}^{n} \delta_{x} \Theta_{i+1 / 2}^{n}+\widetilde{\theta}_{i+1 / 2}^{n} \delta_{x} C_{i+1 / 2}^{n}+R_{u, i+1 / 2}^{n}\right]^{2} \\
& \quad \leq 5 \sum_{i=0}^{M-1} h\left(4 c_{\max }^{2}\left|\delta_{x} \widetilde{\theta}_{i+1 / 2}^{n}\right|^{2}+4 \theta_{\max }^{2}\left|\delta_{x} \widehat{c}_{i+1 / 2}^{\hat{n}}\right|^{2}\right. \\
& \left.\quad+E_{3}^{2}\left|\widetilde{c}_{i+1 / 2}^{n}\right|^{2}+E_{3}^{2}\left|\widetilde{\theta}_{i+1 / 2}^{n}\right|^{2}+\left|R_{u, i+1 / 2}^{n}\right|^{2}\right) \\
& \leq 20 c_{\max }^{2}\left\|\delta_{x} \widetilde{\theta}^{n}\right\|^{2}+20 \theta_{\max }^{2}\left\|\delta_{x} \widehat{c}^{\hat{n}}\right\|^{2}+5 E_{3}^{2}\left(\left\|\widetilde{c}^{n}\right\|^{2}\right. \\
& \left.+\left\|\widetilde{\theta}^{n}\right\|^{2}\right)+5 E_{2}^{2} L\left(\tau^{2}+h^{2}\right)^{2}, \quad 1 \leq n \leq k
\end{aligned}
$$

We can see that, when $\tau \leq h$, the assumption of induction and (68) show that

$$
\begin{aligned}
\left\|\tilde{u}^{\hat{k}}\right\|^{2} & \leq 40 \theta_{\max }^{2} \frac{\left\|\widehat{c}^{k+1}\right\|^{2}}{h^{2}}+E_{c} h^{4} \\
& \leq 160 \theta_{\max }^{2} E_{4} h^{2}+E_{c} h^{4},
\end{aligned}
$$

and when $h \leq \tau$, by (68),

$$
\begin{aligned}
\left\|\tilde{u}^{\hat{k}}\right\|^{2} & \leq 20 \theta_{\max }^{2} \frac{E_{4}\left(\tau^{2}+h^{2}\right)^{2}}{\tau \theta_{\min }}+E_{c} \tau^{4} \\
& \leq 80 \frac{\theta_{\max }^{2}}{\theta_{\min }} E_{4} \tau^{3}+E_{c} \tau^{4},
\end{aligned}
$$

which means there exists an $E_{5}$ independent of $k$, such that

$$
\left\|\tilde{u}^{\hat{k}}\right\| \leq E_{5}\left(\tau^{3 / 2}+h\right) .
$$

Multiplying the error equation (41) by $\delta_{x}^{*} u_{i}^{\widehat{k}}$ leads to

$$
\begin{aligned}
\left\|\delta_{x}^{*} \widetilde{u}^{\hat{k}}\right\|^{2} \leq & \left|\sum_{i=1}^{M-1} \nabla_{t} \vec{c}_{i}^{k} \delta_{x}^{*} u_{i}^{\hat{k}}\right|+\left|\sum_{i=1}^{M-1} \bar{R}_{c, i} \delta_{x}^{*} u_{i}^{\hat{k}}\right| \\
\leq & 4\left\|\nabla_{t} \widetilde{c}^{k}\right\|^{2}+8 E_{3}^{2}\left(\left\|\widetilde{\theta}^{k}\right\|^{2}+\left\|\widetilde{c}^{k}\right\|^{2}\right) \\
& +\frac{3}{4}\left\|\delta_{x}^{*} \widetilde{u}^{\hat{k}}\right\|^{2}+4 E_{2}^{2} L\left(\tau^{2}+h^{2}\right)^{2},
\end{aligned}
$$

that is,

$$
\begin{aligned}
\left\|\delta_{x}^{*} \tilde{u}^{\hat{k}}\right\|^{2} \leq & 16\left\|\nabla_{t} \tilde{c}^{k}\right\|^{2}+32 E_{3}^{2}\left(\left\|\widetilde{\theta}^{k}\right\|^{2}+\left\|\widetilde{c}^{k}\right\|^{2}\right) \\
& +16 E_{2}^{2} L\left(\tau^{2}+h^{2}\right)^{2} .
\end{aligned}
$$

We can see that, when $\tau \leq h$,

$$
\left\|\widetilde{u}^{\hat{k}}\right\|_{\infty} \leq h^{-1 / 2}\left\|\widetilde{u}^{\hat{k}}\right\| \leq E_{5}\left(h+h^{1 / 2}\right),
$$


and when $h \leq \tau$, with Lemma 2 ,

$$
\left\|\tilde{u}^{\hat{k}}\right\|_{\infty}^{2} \leq\left\|\delta_{x}^{*} \tilde{u}^{\hat{k}}\right\|^{2}+\left(1+L^{-1}\right)\left\|\tilde{u}^{\hat{k}}\right\|^{2} \leq E_{6} \tau^{2},
$$

where $E_{6}$ is independent of $k$. Then there exists $s_{0}>0$, when $h, \tau \leq s_{0}$,

$$
\left\|u^{\hat{k}}\right\|_{\infty}=\max _{1 \leq i \leq M}\left|u_{i-1 / 2}^{\hat{k}}\right| \leq 2 E_{3} .
$$

With a time step condition $\tau \leq E_{c} h$, we can see that the coefficient matrix of the system (21)-(23) is strictly diagonally dominant. Thus this system has a unique solution $\theta_{i}^{k+1}$.

3.3. The Optimal Error Estimate. We have proved the existence and uniqueness of the solution to the system and have derived the estimate (65) for $\widetilde{c}^{n+1}$. In this part, we try to derive an estimate for $\widetilde{\theta}^{n+1}$.

Multiplying (45)-(47) by $h \widetilde{\theta}_{i}^{n}, h \widetilde{\theta}_{0}^{n} / 2$, and $h \widetilde{\theta}_{M}^{\hat{n}} / 2$, respectively, we try to estimate each term below:

$$
\begin{aligned}
& h\left[\frac{1}{2}\left(c_{0}^{\widehat{n}}+\sigma\right)\left(\nabla_{t} \widetilde{\theta}_{0}^{n}\right) \widetilde{\theta}_{0}^{\widehat{n}}+\sum_{i=1}^{M-1}\left(c_{i}^{\widehat{n}}+\sigma\right)\left(\nabla_{t} \widetilde{\theta}_{i}^{n}\right) \widetilde{\theta}_{i}^{\widehat{n}}\right. \\
& \left.+\frac{1}{2}\left(c_{M}^{\widehat{n}}+\sigma\right)\left(\nabla_{t} \widetilde{\theta}_{M}^{n}\right) \widetilde{\theta}_{M}^{\widehat{n}}\right]-\frac{h}{2}\left[u_{1 / 2}^{\widehat{n}}\left(\delta_{x} \widetilde{\theta}_{1 / 2}^{\widehat{n}}\right) \widetilde{\theta}_{0}^{n}\right. \\
& +\sum_{i=1}^{M-1}\left(u_{i-1 / 2}^{\widehat{n}} \delta_{x} \widetilde{\theta}_{i-1 / 2}^{\widehat{n}}+u_{i+1 / 2}^{\widehat{n}} \delta_{x} \widetilde{\theta}_{i+1 / 2}^{\widehat{n}}\right) \widetilde{\theta}_{i}^{\widehat{n}} \\
& \left.+u_{M-1 / 2}^{\widehat{n}}\left(\delta_{x} \widetilde{\theta}_{M-1 / 2}^{\widehat{n}}\right) \widetilde{\theta}_{M}^{\widehat{n}}\right]-\kappa\left[\widetilde{w}_{1 / 2}^{\widehat{n}} \widetilde{\theta}_{0}^{\widehat{n}}\right. \\
& \left.+h \sum_{i=1}^{M-1} \delta_{x}^{*} \widetilde{w}_{i+1 / 2}^{\widehat{n}} \widetilde{\theta}_{i}^{\widehat{n}}-\widetilde{w}_{M-1 / 2}^{\widehat{n}} \widetilde{\theta}_{M}^{\widehat{n}}\right]+\kappa \beta_{1}\left|\widehat{\theta}_{0}^{n}\right|^{2} \\
& +\kappa \beta_{2}\left|\widetilde{\theta}_{M}^{\widehat{n}}\right|^{2}=-h\left[\frac{1}{2} \widetilde{c}_{0}^{\widehat{n}}\left(\nabla_{t} \Theta_{0}^{n}\right) \widetilde{\theta}_{0}^{\widehat{n}}\right. \\
& \left.+\sum_{i=1}^{M-1} \widetilde{c}_{i}^{\widehat{n}}\left(\nabla_{t} \Theta_{i}^{n}\right) \widetilde{\theta}_{i}^{\widehat{n}}+\frac{1}{2} \widehat{c}_{M}^{\widehat{n}}\left(\nabla_{t} \Theta_{M}^{n}\right) \widetilde{\theta}_{M}^{\hat{n}}\right] \\
& +\frac{h}{2}\left[\widetilde{u}_{1 / 2}^{\widehat{n}}\left(\delta_{x} \Theta_{1 / 2}^{\widehat{n}}\right) \widetilde{\theta}_{0}^{\widehat{n}}\right. \\
& +\sum_{i=1}^{M-1}\left(\tilde{u}_{i-1 / 2}^{\widehat{n}} \delta_{x} \Theta_{i-1 / 2}^{\widehat{n}}+\widetilde{u}_{i+1 / 2}^{\widehat{n}} \delta_{x} \Theta_{i+1 / 2}^{\widehat{n}}\right) \widetilde{\theta}_{i}^{\widehat{n}} \\
& \left.+\widetilde{u}_{M-1 / 2}^{\widehat{n}}\left(\delta_{x} \Theta_{M-1 / 2}^{\widehat{n}}\right) \widetilde{\theta}_{M}^{\widehat{n}}\right]+\frac{h}{2}\left[\left(\lambda+\Theta_{0}^{n}\right)\right. \\
& \left.\cdot \Gamma\left(C_{0}^{n}, \Theta_{0}^{n}\right)-\left(\lambda+\theta_{0}^{n}\right) \Gamma\left(c_{0}^{n}, \theta_{0}^{n}\right)\right] \widetilde{\theta}_{0}^{n} \\
& +h \sum_{i=1}^{M-1}\left[\left(\lambda+\Theta_{i}^{n}\right) \Gamma\left(C_{i}^{n}, \Theta_{i}^{n}\right)-\left(\lambda+\theta_{i}^{n}\right) \Gamma\left(c_{i}^{n}, \theta_{i}^{n}\right)\right]
\end{aligned}
$$

$$
\begin{aligned}
& \cdot \widetilde{\theta}_{i}^{\hat{n}}+\frac{h}{2}\left[\left(\lambda+\Theta_{M}^{n}\right) \Gamma\left(C_{M}^{n}, \Theta_{M}^{n}\right)-\left(\lambda+\theta_{M}^{n}\right)\right. \\
& \left.\cdot \Gamma\left(c_{M}^{n}, \theta_{M}^{n}\right)\right] \widetilde{\theta}_{M}^{\widehat{n}}+\left[h \sum_{i=1}^{M-1} R_{\theta, i}^{n} \widetilde{\theta}_{i}^{\widehat{n}}+\frac{h}{2} R_{\theta, 0}^{n} \widetilde{\theta}_{0}^{\widehat{n}}+\frac{h}{2}\right. \\
& \left.\cdot R_{\theta, M}^{n} \widetilde{\theta}_{M}^{n}\right]:=J_{3}+J_{4}+J_{5}+J_{6}, \quad 1 \leq n \leq k .
\end{aligned}
$$

According to Lemma 2, (40), (51), and (60), three terms on the left can be bounded by

$$
\begin{aligned}
& h\left[\frac{1}{2}\left(c_{0}^{\widehat{n}}+\sigma\right)\left(\nabla_{t} \widetilde{\theta}_{0}^{n}\right) \widetilde{\theta}_{0}^{\widehat{n}}+\sum_{i=1}^{M-1}\left(c_{i}^{\widehat{n}}+\sigma\right)\left(\nabla_{t} \widetilde{\theta}_{i}^{n}\right) \widetilde{\theta}_{i}^{\widehat{n}}\right. \\
& \left.+\frac{1}{2}\left(c_{M}^{\widehat{n}}+\sigma\right)\left(\nabla_{t} \widetilde{\theta}_{M}^{n}\right) \widetilde{\theta}_{M}^{\widehat{n}}\right]=\frac{h}{2}\left\{\frac{1}{2}\left(c_{0}^{\widehat{n}}+\sigma\right)\right. \\
& \cdot \nabla_{t}\left[\left(\widetilde{\theta}_{0}^{n}\right)^{2}\right]+\sum_{i=1}^{M-1}\left(c_{i}^{\widehat{n}}+\sigma\right) \nabla_{t}\left[\left(\widetilde{\theta}_{i}^{n}\right)^{2}\right]+\frac{1}{2}\left(c_{M}^{\widehat{n}}\right. \\
& \left.+\sigma) \nabla_{t}\left[\left(\widetilde{\theta}_{M}^{n}\right)^{2}\right]\right\} \\
& \mid-\frac{h}{2}\left[u_{1 / 2}^{\widehat{n}}\left(\delta_{x} \widetilde{\theta}_{1 / 2}^{\widehat{n}}\right) \widetilde{\theta}_{0}^{\underline{n}}\right. \\
& +\sum_{i=1}^{M-1}\left(u_{i-1 / 2}^{\widehat{n}} \delta_{x} \widetilde{\theta}_{i-1 / 2}^{\widehat{n}}+u_{i+1 / 2}^{\widehat{n}} \delta_{x} \widetilde{\theta}_{i+1 / 2}^{\widehat{n}}\right) \widetilde{\theta}_{i}^{\widehat{n}} \\
& \left.+u_{M-1 / 2}^{\widehat{n}}\left(\delta_{x} \widetilde{\theta}_{M-1 / 2}^{\widehat{n}}\right) \widetilde{\theta}_{M}^{\widehat{n}}\right] \mid \\
& =\left|-h \sum_{i=0}^{M-1} u_{i+1 / 2}^{\widehat{n}} \delta_{x} \widetilde{\theta}_{i+1 / 2}^{\widehat{n}} \widetilde{\theta}_{i+1 / 2}^{\widehat{n}}\right| \\
& \leq 2 E_{3}\left|h \sum_{i=0}^{M-1} \delta_{x} \widetilde{\theta}_{i+1 / 2}^{\widehat{n}} \widetilde{\theta}_{i+1 / 2}^{\widehat{n}}\right| \leq \frac{\kappa}{4}\left\|\delta_{x} \widetilde{\theta}^{\widehat{n}}\right\|^{2}+E_{c}\left\|\widetilde{\theta}^{\widehat{n}}\right\|^{2},
\end{aligned}
$$

and

$$
\begin{aligned}
& -\kappa\left[\widetilde{w}_{1 / 2}^{\widehat{n}} \widetilde{\theta}_{0}^{\widehat{n}}+h \sum_{i=1}^{M-1} \delta_{x}^{*} \widetilde{w}_{i}^{\widehat{n}} \widetilde{\theta}_{i}^{\widehat{n}}-\widetilde{w}_{M-1 / 2}^{\widehat{n}} \widetilde{\theta}_{M}^{n}\right] \\
& =\kappa h \sum_{i=0}^{M-1}\left(\delta_{x} \widetilde{\theta}_{i+1 / 2}^{\hat{n}}\right)^{2}+\kappa h \sum_{i=0}^{M-1} R_{w, i+1 / 2} \delta_{x} \widetilde{\theta}_{i+1 / 2}^{\widehat{n}} \\
& \geq \kappa\left\|\delta_{x} \widetilde{\theta}^{\widehat{n}}\right\|^{2}-E_{c} h \sum_{i=0}^{M-1}\left|R_{w, i+1 / 2}^{n}\right|^{2}-\frac{\kappa}{4}\left\|\delta_{x} \widetilde{\theta}^{\widehat{n}}\right\|^{2} \\
& \geq \frac{3 \kappa}{4}\left\|\delta_{x} \widetilde{\theta}^{\hat{n}}\right\|^{2}-E_{c}\left(\tau^{2}+h^{2}\right)^{2}, \quad 1 \leq n \leq k .
\end{aligned}
$$

By (70), for those terms in the right hand side, we obtain

$$
\begin{aligned}
& \left|J_{3}\right| \leq E_{3}\left(\left\|\widehat{c}^{\widehat{n}}\right\|^{2}+\left\|\widetilde{\theta}^{\hat{n}}\right\|^{2}\right), \\
& \left|J_{4}\right| \leq h E_{3}\left(\left|\widehat{u}_{1 / 2}^{\widehat{n}}\right| \cdot\left|\widetilde{\theta}_{0}^{\hat{n}}\right|+\sum_{i=1}^{M-1}\left(\left|\tilde{u}_{i-1 / 2}^{\widehat{\hat{n}}}\right|+\left|\widetilde{u}_{i+1 / 2}^{\hat{n}}\right|\right)\left|\widetilde{\theta}_{i}^{\hat{n}}\right|\right.
\end{aligned}
$$




$$
\begin{aligned}
& \left.+\left|\widetilde{u}_{M-1 / 2}^{\widehat{n}}\right| \cdot\left|\widetilde{\theta}_{M}^{\hat{n}}\right|\right) \leq 2 E_{3}\left(\left\|\widetilde{\theta}^{\hat{n}}\right\|^{2}\right. \\
& \left.+\sum_{i=0}^{M-1} h\left|\widetilde{u}_{i+1 / 2}^{\widehat{n}}\right|^{2}\right) \leq 40 E_{3} \theta_{\max }^{2}\left\|\delta_{x} \widehat{c}^{\hat{c}}\right\|^{2} \\
& +E_{c}\left(\left\|\delta_{x} \widetilde{\theta}^{n}\right\|^{2}+\left\|\widetilde{\theta}^{n}\right\|+\left\|c^{n}\right\|^{2}\right)+E_{2}\left(\tau^{2}+h^{2}\right)^{2}, \\
& \left|J_{5}\right| \leq E_{3}\left(\left\|\widetilde{c}^{n}\right\|+\left\|\widetilde{\theta}^{n}\right\|\right)\left\|\widetilde{\theta}^{\widehat{n}}\right\| . \\
& \left|J_{6}\right| \leq \frac{1}{2} h \sum_{i=1}^{M-1}\left[\left(R_{\theta, i}^{n}\right)^{2}+\left(\widetilde{\theta}_{i}^{\widehat{n}}\right)^{2}\right]+\frac{h^{2}}{2}\left|R_{\theta, 0}^{n}\right|^{2}+\frac{1}{2}\left|\widetilde{\theta}_{0}^{n}\right|^{2} \\
& +\frac{h^{2}}{2}\left|R_{\theta, M}^{n}\right|^{2}+\frac{1}{2}\left|\widetilde{\theta}_{M}^{\hat{n}}\right|^{2} \leq\left\|\widetilde{\theta}^{\hat{n}}\right\|_{\infty}^{2}+E_{c}\left\|\widetilde{\theta}^{\widehat{n}}\right\|^{2} \\
& +\frac{E_{2} L}{2}\left(\tau^{2}+h^{2}\right)^{2}+E_{2} h^{2}\left(\tau^{2}+h\right)^{2} \leq \frac{\kappa}{4}\left\|\delta_{x} \widetilde{\theta}^{\hat{n}}\right\|^{2} \\
& +\left\|\widetilde{\theta}^{\hat{n}}\right\|^{2}+E_{c}\left(\tau^{2}+h^{2}\right)^{2} .
\end{aligned}
$$

Taking the last six equations into (79), we obtain

$$
\begin{aligned}
& \frac{h}{2}\left\{\frac{1}{2}\left(c_{0}^{\widehat{n}}+\sigma\right) \nabla_{t}\left[\left(\widetilde{\theta}_{0}^{n}\right)^{2}\right]+\sum_{i=1}^{M-1}\left(c_{i}^{\widehat{n}}+\sigma\right) \nabla_{t}\left[\left(\widetilde{\theta}_{i}^{n}\right)^{2}\right]\right. \\
& \left.\quad+\frac{1}{2}\left(c_{M}^{\widehat{n}}+\sigma\right) \nabla_{t}\left[\left(\widetilde{\theta}_{M}^{n}\right)^{2}\right]\right\}+\frac{\kappa}{4}\left\|\delta_{x} \widetilde{\theta}^{\hat{n}}\right\|^{2} \\
& \quad+\kappa \beta_{1}\left|\widetilde{\theta}_{0}^{n}\right|^{2}+\kappa \beta_{2}\left|\widetilde{\theta}_{M}^{\hat{n}}\right|^{2} \leq 40 E_{3} \theta_{\max }^{2}\left\|\delta_{x} \widehat{c}^{\widehat{n}}\right\|^{2} \\
& \quad+E_{c}\left(\left\|\delta_{x} \widetilde{\theta}^{n}\right\|^{2}+\left\|\widetilde{c}^{n}\right\|^{2}+\left\|\widetilde{\theta}^{n}\right\|^{2}+\left\|\widehat{c}^{\hat{c}}\right\|^{2}+\left\|\widetilde{\theta}^{\hat{n}}\right\|^{2}\right) \\
& \quad+E_{c}\left(\tau^{2}+h^{2}\right)^{2}, \quad 1 \leq n \leq k .
\end{aligned}
$$

Besides, we introduce a notation as $\widetilde{\theta}_{i}^{*}=(1 / 2)\left[\left(\widetilde{\theta}_{i}^{n+1}\right)^{2}+\right.$ $\left.\left(\widetilde{\theta}_{i}^{n-1}\right)^{2}\right]$, and adding the first three equations into (36) by $h \widetilde{\theta}_{i}^{*} / 2, h \widetilde{\theta}_{0}^{*} / 4$, and $h \widetilde{\theta}_{M}^{*} / 4$, respectively, we have

$$
\begin{aligned}
\frac{h}{2}[ & \left.\frac{1}{2}\left(\nabla_{t} c_{0}^{n}\right) \widetilde{\theta}_{0}^{*}+\sum_{i=1}^{M-1}\left(\nabla_{t} c_{i}^{n}\right) \widetilde{\theta}_{i}^{*}+\frac{1}{2}\left(\nabla_{t} c_{M}^{n}\right) \widetilde{\theta}_{M}^{*}\right] \\
& -\frac{1}{2}\left[u_{1 / 2}^{\widehat{n}} \widetilde{\theta}_{0}^{*}+h \sum_{i=1}^{M-1} \delta_{x}^{*} u_{i+1 / 2}^{\widehat{n}} \widetilde{\theta}_{i}^{*}-u_{M-1 / 2}^{\widehat{n}} \widetilde{\theta}_{M}^{*}\right] \\
& +\frac{\alpha_{1}}{2}\left(c_{0}^{\widehat{n}}-\mu_{1}\right) \widetilde{\theta}_{0}^{*}+\frac{\alpha_{2}}{2}\left(c_{M}^{\widehat{n}}-\mu_{2}\right) \widetilde{\theta}_{M}^{*} \\
= & -\frac{h}{2}\left[\frac{1}{2} \widetilde{\theta}_{0}^{*} \Gamma\left(c_{0}^{n}, \theta_{0}^{n}\right)+\sum_{i=1}^{M-1} \widetilde{\theta}_{i}^{*} \Gamma\left(c_{i}^{n}, \theta_{i}^{n}\right)\right. \\
& \left.+\frac{1}{2} \widetilde{\theta}_{M}^{*} \Gamma\left(c_{M}^{n}, \theta_{M}^{n}\right)\right], \quad 1 \leq n \leq k .
\end{aligned}
$$

We now estimate the terms in (84). By (51), we denote

$$
\begin{aligned}
& \left|J_{7}\right|:=\left|-\frac{1}{2}\left[u_{1 / 2}^{\widehat{n}} \widetilde{\theta}_{0}^{*}+h \sum_{i=1}^{M-1} \delta_{x}^{*} u_{i+1 / 2}^{\widehat{\hat{\theta}}} \widetilde{\theta}_{i}^{*}-u_{M-1 / 2}^{\widehat{\hat{n}}} \widetilde{\theta}_{M}^{*}\right]\right| \\
& =\left|\frac{1}{2} \sum_{i=0}^{M-1} u_{i+1 / 2}^{\widehat{n}}\left(\widetilde{\theta}_{i+1}^{*}-\widetilde{\theta}_{i}^{*}\right)\right| \\
& \leq\left|E_{3} h \sum_{i=0}^{M-1}\left(\widetilde{\theta}_{i+1 / 2}^{n+1} \delta_{x} \widetilde{\theta}_{i+1 / 2}^{n+1}+\widetilde{\theta}_{i+1 / 2}^{n-1} \delta_{x} \widetilde{\theta}_{i+1 / 2}^{n-1}\right)\right| \\
& \leq \frac{E_{3}}{2}\left(\left\|\delta_{x} \widetilde{\theta}^{n+1}\right\|^{2}+\left\|\delta_{x} \widetilde{\theta}^{n-1}\right\|^{2}+\left\|\widetilde{\theta}^{n+1}\right\|^{2}+\left\|\widetilde{\theta}^{n-1}\right\|^{2}\right) .
\end{aligned}
$$

Using (60) again, we get

$$
\begin{aligned}
\mid-\frac{h}{2}\left[\frac{1}{2} \widetilde{\theta}_{0}^{*} \Gamma\left(c_{0}^{n}, \theta_{0}^{n}\right)+\sum_{i=1}^{M-1} \widetilde{\theta}_{i}^{*} \Gamma\left(c_{i}^{n}, \theta_{i}^{n}\right)\right. \\
\left.+\frac{1}{2} \widetilde{\theta}_{M}^{*} \Gamma\left(c_{M}^{n}, \theta_{M}^{n}\right)\right] \mid \leq \frac{E_{3}}{4}\left(\left\|\widetilde{\theta}^{n+1}\right\|^{2}+\left\|\widetilde{\theta}^{n-1}\right\|^{2}\right),
\end{aligned}
$$

and with Lemma 2, we have

$$
\begin{aligned}
& \frac{h}{2}\left[\frac{1}{2}\left(\nabla_{t} c_{0}^{n}\right) \widetilde{\theta}_{0}^{*}+\sum_{i=1}^{M-1}\left(\nabla_{t} c_{i}^{n}\right) \widetilde{\theta}_{i}^{*}+\frac{1}{2}\left(\nabla_{t} c_{M}^{n}\right) \widetilde{\theta}_{M}^{*}\right] \\
& \leq \frac{\alpha_{1} \mu_{1}}{2} \widetilde{\theta}_{0}^{*}+\frac{\alpha_{2} \mu_{2}}{2} \widetilde{\theta}_{M}^{*} \\
& \quad+E_{c}\left(\left\|\widetilde{\theta}^{n+1}\right\|^{2}+\left\|\widetilde{\theta}^{n-1}\right\|^{2}+\left\|\delta_{x} \widetilde{\theta}^{n+1}\right\|^{2}+\left\|\delta_{x} \widetilde{\theta}^{n-1}\right\|^{2}\right) \\
& \leq \frac{\alpha_{1} \mu_{1}+\alpha_{2} \mu_{2}}{2}\left(\left\|\widetilde{\theta}^{n+1}\right\|_{\infty}^{2}+\left\|\widetilde{\theta}^{n-1}\right\|_{\infty}^{2}\right) \\
& \quad+E_{c}\left(\left\|\widetilde{\theta}^{n+1}\right\|^{2}+\left\|\widetilde{\theta}^{n-1}\right\|^{2}+\left\|\delta_{x} \widetilde{\theta}^{n+1}\right\|^{2}+\left\|\delta_{x} \widetilde{\theta}^{n-1}\right\|^{2}\right) \\
& \leq E_{c}\left(\left\|\widetilde{\theta}^{n+1}\right\|^{2}+\left\|\widetilde{\theta}^{n-1}\right\|^{2}+\left\|\delta_{x} \widetilde{\theta}^{n+1}\right\|^{2}+\left\|\delta_{x} \widetilde{\theta}^{n-1}\right\|^{2}\right) .
\end{aligned}
$$

Moreover, by noting the fact that

$$
\left(c_{i}^{\widehat{n}}+\sigma\right) \nabla_{t}\left[\left(\widetilde{\theta}_{i}^{n}\right)^{2}\right]+\widetilde{\theta}_{i}^{*} \nabla_{t} c_{i}^{n}=\nabla_{t}\left[\left(c_{i}^{n}+\sigma\right)\left(\widetilde{\theta}_{i}^{n}\right)^{2}\right],
$$

adding (87) in (84) and using Lemma 2 again, we further get

$$
\begin{aligned}
& \frac{h}{2}\left\{\frac{1}{2} \nabla_{t}\left[\left(c_{0}^{n}+\sigma\right)\left(\widetilde{\theta}_{0}^{n}\right)^{2}\right]+\sum_{i=1}^{M-1} \nabla_{t}\left[\left(c_{i}^{n}+\sigma\right)\left(\widetilde{\theta}_{i}^{n}\right)^{2}\right]\right. \\
& \left.\quad+\frac{1}{2} \nabla_{t}\left[\left(c_{M}^{n}+\sigma\right)\left(\widetilde{\theta}_{M}^{n}\right)^{2}\right]\right\}+\frac{\kappa}{4}\left\|\delta_{x} \widetilde{\theta}^{\hat{n}}\right\|^{2}+\kappa \beta_{1}\left|\widetilde{\theta}_{0}^{n}\right|^{2} \\
& +\kappa \beta_{2}\left|\widetilde{\theta}_{M}^{n}\right|^{2} \leq 40 E_{3} \theta_{\max }^{2}\left\|\delta_{x} \widehat{c}^{\widehat{c}}\right\|^{2}+E_{c}\left(\left\|\delta_{x} \widetilde{\theta}^{n}\right\|^{2}\right. \\
& \quad+\left\|\widetilde{\theta}^{n+1}\right\|^{2}+\left\|\widetilde{c}^{n+1}\right\|^{2}+\left\|\widetilde{\theta}^{n}\right\|^{2}+\left\|\widetilde{c}^{n}\right\|^{2}+\left\|\widetilde{\theta}^{n-1}\right\|^{2} \\
& \left.\quad+\left\|\widetilde{c}^{n-1}\right\|^{2}\right)+E_{c}\left(\tau^{2}+h^{2}\right)^{2}, \quad 1 \leq n \leq k .
\end{aligned}
$$


Multiplying the last equation with $s_{1}=\theta_{\min } / 320 E_{3} \theta_{\max }^{2}$ and adding it into equation (65), we get

$$
\begin{aligned}
\nabla_{t} & \left\|\widetilde{c}^{n}\right\|^{2}+\frac{\theta_{\min }}{8}\left\|\delta_{x} \widetilde{c}^{\hat{n}}\right\|^{2}+\left[2 \alpha_{1}\left|\widehat{c}_{0}\right|^{2}+2 \alpha_{2}\left|\widehat{c}_{M}^{\widehat{n}}\right|^{2}\right. \\
& \left.+s_{1} \kappa \beta_{1}\left|\widetilde{\theta}_{0}^{n}\right|^{2}+s_{1} \kappa \beta_{2}\left|\widetilde{\theta}_{M}^{n}\right|^{2}\right] \\
& +\frac{s_{1} h}{2}\left[\frac{1}{2} \nabla_{t}\left[\left(c_{0}^{n}+\sigma\right)\left|\widetilde{\theta}_{0}^{n}\right|^{2}\right]\right. \\
& \left.+\sum_{i=1}^{M-1} \nabla_{t}\left[\left(c_{i}^{n}+\sigma\right)\left|\widetilde{\theta}_{i}^{n}\right|^{2}\right]+\frac{1}{2} \nabla_{t}\left[\left(c_{M}^{n}+\sigma\right)\left|\widetilde{\theta}_{M}^{n}\right|^{2}\right]\right] \\
& +\frac{\kappa s_{1}}{4}\left\|\delta_{x} \widetilde{\theta}^{n}\right\|^{2} \leq E_{c}\left(\left\|\delta_{x} \widetilde{\theta}^{n}\right\|^{2}+\left\|\widetilde{\theta}^{n+1}\right\|^{2}+\left\|\widetilde{c}^{n+1}\right\|^{2}\right. \\
& \left.+\left\|\widetilde{\theta}^{n}\right\|^{2}+\left\|\widetilde{c}^{n}\right\|^{2}+\left\|\widetilde{\theta}^{n-1}\right\|^{2}+\left\|\widetilde{c}^{n-1}\right\|^{2}\right)+E_{c}\left(\tau^{2}\right. \\
& \left.+h^{2}\right)^{2}, \quad 1 \leq n \leq k .
\end{aligned}
$$

Finally we estimate $\left\|\delta_{x} \widetilde{\theta}^{n}\right\|$. Multiplying the error equation (45) by $-h \delta_{x}^{2} \widetilde{\theta}^{\hat{n}} /\left(c_{i}^{\widehat{n}}+\sigma\right)$ and summing up the resulting equations for $i=1,2, \ldots, M-1$, we have

$$
\begin{aligned}
- & h \sum_{i=1}^{M-1}\left(\nabla_{t} \widetilde{\theta}_{i}^{n}\right) \delta_{x}^{2} \widetilde{\theta}_{i}^{\hat{n}}+\frac{\kappa}{c_{i}^{\widehat{n}}+\sigma} h \sum_{i=1}^{M-1} \delta_{x}^{*} \widetilde{w}_{i}^{\widehat{n}} \delta_{x}^{2} \widetilde{\theta}_{i}^{\hat{n}}=\frac{h}{c_{i}^{\widehat{n}}+\sigma} \\
& \cdot \sum_{i=1}^{M-1} \widetilde{c}_{i}^{\widehat{n}}\left(\nabla_{t} \Theta_{i}^{n}\right) \delta_{x}^{2} \widetilde{\theta}_{i}^{\widehat{n}}-\frac{h}{2\left(c_{i}^{\widehat{n}}+\sigma\right)} \\
& \cdot \sum_{i=1}^{M-1}\left(\widetilde{u}_{i-1 / 2}^{\widehat{n}} \delta_{x} \Theta_{i-1 / 2}^{\widehat{n}}+\widetilde{u}_{i+1 / 2}^{\widehat{n}} \delta_{x} \Theta_{i+1 / 2}^{\widehat{n}}\right) \delta_{x}^{2} \widetilde{\theta}_{i}^{\hat{n}} \\
& -\frac{h}{c_{i}^{\widehat{n}}+\sigma} \sum_{i=1}^{M-1}\left[\left(\lambda+\Theta_{i}^{n}\right) \Gamma\left(C_{i}^{n}, \Theta_{i}^{n}\right)\right. \\
& \left.-\left(\lambda+\theta_{i}^{n}\right) \Gamma\left(c_{i}^{n}, \theta_{i}^{n}\right)+R_{\theta, i}^{n}\right] \delta_{x}^{2} \widetilde{\theta}_{i}^{\hat{n}}-\frac{h}{2\left(c_{i}^{\widehat{n}}+\sigma\right)} \\
& \cdot \sum_{i=1}^{M-1}\left(u_{i-1 / 2}^{\widehat{n}} \delta_{x} \widetilde{\theta}_{i-1 / 2}^{\hat{n}}+u_{i+1 / 2}^{\widehat{n}} \delta_{x} \widetilde{\theta}_{i+1 / 2}^{\widehat{n}}\right) \delta_{x}^{2} \widetilde{\theta}_{i}^{\hat{n}},
\end{aligned}
$$

$1 \leq n \leq k$.

For the first term, we have

$$
\begin{aligned}
- & h \sum_{i=1}^{M-1}\left(\nabla_{t} \widetilde{\theta}_{i}^{n}\right) \delta_{x}^{2} \widetilde{\theta}_{i}^{\widehat{n}} \\
= & -\frac{h}{2 \tau} \sum_{i=1}^{M-1} \widetilde{\theta}_{i}^{n+1} \delta_{x}^{2} \widetilde{\theta}_{i}^{n}+\frac{h}{2 \tau} \sum_{i=1}^{M-1} \widetilde{\theta}_{i}^{n-1} \delta_{x}^{2} \widetilde{\theta}_{i}^{\hat{n}} \\
= & -\frac{1}{2 \tau} \sum_{i=1}^{M-1} \widetilde{\theta}_{i}^{n+1}\left(\delta_{x} \widetilde{\theta}_{i+1 / 2}^{n}-\delta_{x} \widetilde{\theta}_{i-1 / 2}^{n}\right) \\
& +\frac{1}{2 \tau} \sum_{i=1}^{M-1} \widetilde{\theta}_{i}^{n-1}\left(\delta_{x} \widetilde{\theta}_{i+1 / 2}^{n}-\delta_{x} \widetilde{\theta}_{i-1 / 2}^{n}\right) .
\end{aligned}
$$

By (51), we have

$$
\begin{aligned}
& -h \sum_{i=1}^{M-1}\left(\nabla_{t} \widetilde{\theta}_{i}^{n}\right) \delta_{x}^{2} \widetilde{\theta}_{i}^{n}=-\frac{1}{2 \tau}\left(\widetilde{\theta}_{M}^{n+1} \delta_{x} \widetilde{\theta}_{M-1 / 2}^{n}\right. \\
& \left.-\widetilde{\theta}_{1}^{n+1} \delta_{x} \widetilde{\theta}_{1 / 2}^{n}-\sum_{i=1}^{M-1} \delta_{x} \widetilde{\theta}_{i+1 / 2}^{n}\left(\widetilde{\theta}_{i+1}^{n+1}-\widetilde{\theta}_{i}^{n+1}\right)\right) \\
& +\frac{1}{2 \tau}\left(\widetilde{\theta}_{M}^{n-1} \delta_{x} \widetilde{\theta}_{M-1 / 2}^{n}-\widetilde{\theta}_{1}^{n-1} \delta_{x} \widetilde{\theta}_{1 / 2}^{n}\right. \\
& \left.-\sum_{i=1}^{M-1} \delta_{x} \widetilde{\theta}_{i+1 / 2}^{n}\left(\widetilde{\theta}_{i+1}^{n-1}-\widetilde{\theta}_{i+1}^{n-1}\right)\right)=-\nabla_{t} \widetilde{\theta}_{M}^{n} \delta_{x} \widetilde{\theta}_{M-1 / 2}^{n} \\
& +\nabla_{t} \widetilde{\theta}_{1}^{n} \delta_{x} \widetilde{\theta}_{1 / 2}^{n}+\frac{1}{4 \tau}\left(h \sum_{i=1}^{M-1}\left(\delta_{x} \widetilde{\theta}_{i+1 / 2}^{n+1}\right)^{2}\right. \\
& \left.-h \sum_{i=1}^{M-1}\left(\delta_{x} \widetilde{\theta}_{i+1 / 2}^{n-1}\right)^{2}\right)=\frac{1}{2} \nabla_{t}\left\|\delta_{x} \widetilde{\theta}^{n}\right\|^{2} \\
& -\nabla_{t} \widetilde{\theta}_{M}^{n} \delta_{x} \widetilde{\theta}_{M-1 / 2}^{n}+\nabla_{t} \widetilde{\theta}_{0}^{n} \delta_{x} \widetilde{\theta}_{1 / 2}^{n} .
\end{aligned}
$$

For the second term, we have

$$
\begin{aligned}
& \frac{\kappa}{c_{i}^{\widehat{n}}+\sigma} h \sum_{i=1}^{M-1} \delta_{x}^{*} \widetilde{w}_{i}^{\widehat{n}} \delta_{x}^{2} \widetilde{\theta}_{i}^{\hat{n}} \\
& \geq \frac{\kappa}{c_{\max }+\sigma} h \sum_{i=1}^{M-1}\left|\delta_{x}^{2} \widetilde{\theta}_{i}^{\widehat{n}}\right|^{2} \\
& \quad+\frac{\kappa}{c_{\max }+\sigma} h \sum_{i=1}^{M-1} \delta_{x}^{*} R_{w, i}^{n} \delta_{x}^{2} \widetilde{\theta}_{i}^{\hat{n}} \\
& \geq \frac{\kappa}{2\left(c_{\max }+\sigma\right)} h \sum_{i=1}^{M-1}\left|\delta_{x}^{2} \widetilde{\theta}_{i}^{\hat{n}}\right|^{2}-E_{c}\left(\tau^{2}+h^{2}\right)^{2},
\end{aligned}
$$

where we noted the fact that $\left|\delta_{x}^{*} R_{w, i}^{n}\right| \leq E_{c}\left(\tau^{2}+h^{2}\right)$. From (94) we can get

$$
\begin{gathered}
\frac{1}{2} \nabla_{t}\left\|\delta_{x} \widetilde{\theta}^{n}\right\|^{2}+\frac{\kappa}{2\left(c_{\max }+\sigma\right)}\left\|\delta_{x}^{2} \widetilde{\theta}^{\hat{n}}\right\|^{2}+\nabla_{t} \widetilde{\theta}_{0}^{n} \delta_{x} \widetilde{\theta}_{1 / 2}^{\hat{n}} \\
-\nabla_{t} \widetilde{\theta}_{M}^{n} \delta_{x} \widetilde{\theta}_{M-1 / 2}^{\hat{n}} \leq \frac{h}{c_{i}^{\widehat{n}}+\sigma} \sum_{i=1}^{M-1} \widetilde{c}_{i}^{\widehat{n}}\left(\nabla_{t} \Theta_{i}^{n}\right) \delta_{x}^{2} \widetilde{\theta}_{i}^{\hat{n}} \\
-\frac{h}{2\left(c_{i}^{\widehat{n}}+\sigma\right)} \sum_{i=1}^{M-1}\left(\widetilde{u}_{i-1 / 2}^{\widehat{n}} \delta_{x} \Theta_{i-1 / 2}^{\widehat{n}}+\widetilde{u}_{i+1 / 2}^{\widehat{n}} \delta_{x} \Theta_{i+1 / 2}^{\widehat{n}}\right) \\
\cdot \delta_{x}^{2} \widetilde{\theta}_{i}^{n}-\frac{h}{c_{i}^{\widehat{n}}+\sigma} \sum_{i=1}^{M-1}\left[\left(\lambda+\Theta_{i}^{n}\right) \Gamma\left(C_{i}^{n}, \Theta_{i}^{n}\right)\right. \\
\left.-\left(\lambda+\theta_{i}^{n}\right) \Gamma\left(c_{i}^{n}, \theta_{i}^{n}\right)+R_{\theta, i}^{n}\right] \delta_{x}^{2} \widetilde{\theta}_{i}^{\widehat{n}}-\frac{h}{2\left(c_{i}^{\widehat{n}}+\sigma\right)}
\end{gathered}
$$




$$
\begin{aligned}
& \cdot \sum_{i=1}^{M-1}\left(u_{i-1 / 2}^{\widehat{n}} \delta_{x} \widetilde{\theta}_{i-1 / 2}^{\hat{n}}+u_{i+1 / 2}^{\widehat{n}} \delta_{x} \widetilde{\theta}_{i+1 / 2}^{\hat{n}}\right) \delta_{x}^{2} \widetilde{\theta}_{i}^{\hat{n}} \\
& +E_{c}\left(\tau^{2}+h^{2}\right)^{2}:=J_{8}+J_{9}+J_{10}+J_{11}, \quad 1 \leq n \leq k .
\end{aligned}
$$

Then we estimate the term $\nabla_{t} \widetilde{\theta}_{0}^{n} \delta_{x} \widetilde{\theta}_{1 / 2}^{n},-\nabla_{t} \widetilde{\theta}_{M}^{n} \delta_{x} \widetilde{\theta}_{M-1 / 2}^{n}$ and $J_{8}$ to $J_{11}$, respectively. From (46), we have

$$
\begin{aligned}
\delta_{x} \widetilde{\theta}_{1 / 2}^{n}= & \beta_{1} \widetilde{\theta}_{0}^{\widehat{n}} \\
& +\frac{h}{2 \kappa}\left[\left(c_{0}^{\widehat{n}}+\sigma\right) \nabla_{t} \widetilde{\theta}_{0}^{n}-u_{1 / 2}^{\widehat{n}} \delta_{x} \widetilde{\theta}_{1 / 2}^{n}-\vec{R}_{\theta, 0}^{n}\right] \\
& -R_{w, 1 / 2}^{n} .
\end{aligned}
$$

A straightforward calculation with Lemma 2 leads to

$$
\begin{aligned}
& \nabla_{t} \widetilde{\theta}_{0}^{n} \delta_{x} \widetilde{\theta}_{1 / 2}^{\widehat{n}}=\beta_{1} \nabla_{t} \widetilde{\theta}_{0}^{n} \widetilde{\theta}_{0}^{\widehat{n}}+\frac{h}{2 \kappa}\left[\left(c_{0}^{\widehat{n}}+\sigma\right) \nabla_{t} \widetilde{\theta}_{0}^{n}\right. \\
& \left.-u_{1 / 2}^{\widehat{n}} \delta_{x} \widetilde{\theta}_{1 / 2}^{n}-\bar{R}_{\theta, 0}^{n}\right] \nabla_{t} \widetilde{\theta}_{0}^{n}-R_{w, 1 / 2}^{n} \nabla_{t} \widetilde{\theta}_{0}^{n} \geq \frac{\beta_{1}}{2} \\
& \cdot \nabla_{t}\left(\left|\widetilde{\theta}_{0}^{n}\right|^{2}\right)+\frac{h \sigma}{2 \kappa}\left|\nabla_{t} \widetilde{\theta}_{0}^{n}\right|^{2}-\frac{h}{2 \kappa} u_{1 / 2}^{\widehat{n}} \delta_{x} \widetilde{\theta}_{1 / 2}^{n} \nabla_{t} \widetilde{\theta}_{0}^{n} \\
& -\frac{h}{2 \kappa} \bar{R}_{\theta, 0}^{n} \nabla_{t} \widetilde{\theta}_{0}^{n}-\nabla_{t}\left(R_{w, 1 / 2}^{n} \widetilde{\theta}_{0}^{n}\right)+\frac{R_{w, 1 / 2}^{n+1}-R_{w, 1 / 2}^{n}}{2 \tau} \\
& \cdot \widetilde{\theta}_{0}^{n+1}+\frac{R_{w, 1 / 2}^{n}-R_{w, 1 / 2}^{n-1}}{2 \tau} \widetilde{\theta}_{0}^{n-1} \geq \frac{\beta_{1}}{2} \nabla_{t}\left(\left|\widetilde{\theta}_{0}^{n}\right|^{2}\right) \\
& +\frac{h \sigma}{4 \kappa}\left|\nabla_{t} \widetilde{\theta}_{0}^{n}\right|^{2}-\frac{h}{2 \kappa \sigma}\left[\left|u_{1 / 2}^{\widehat{n}} \delta_{x} \widetilde{\theta}_{1 / 2}^{n}\right|^{2}+\left|\bar{R}_{\theta, 0}^{n}\right|^{2}\right] \\
& -\nabla_{t}\left(R_{w, 1 / 2}^{n} \widetilde{\theta}_{0}^{n}\right)-E_{c}\left(\tau^{2}+h^{2}\right)\left(\left\|\widetilde{\theta}^{n+1}\right\|_{\infty}\right. \\
& \left.+\left\|\widetilde{\theta}^{n-1}\right\|_{\infty}\right) \geq \frac{\beta_{1}}{2} \nabla_{t}\left(\left|\widetilde{\theta}_{0}^{n}\right|^{2}\right)+\frac{h \sigma}{4 \kappa}\left|\nabla_{t} \widetilde{\theta}_{0}^{n}\right|^{2} \\
& -\nabla_{t}\left(R_{w, 1 / 2}^{n} \widetilde{\theta}_{0}^{n}\right)-\frac{2 E_{3}^{2}}{\kappa \sigma}\left\|\delta_{x} \widetilde{\theta}^{n}\right\|^{2} \\
& -\frac{50 E_{3}^{2} \theta_{\max }^{2}}{\kappa \sigma}\left\|\delta_{x} \widehat{c}^{\widehat{\imath}}\right\|^{2}-E_{c}\left(\left\|\widetilde{\theta}^{n+1}\right\|^{2}+\left\|\widetilde{\theta}^{n-1}\right\|^{2}\right. \\
& +\left\|\delta_{x} \widetilde{\theta}^{n+1}\right\|^{2}+\left\|\delta_{x} \widetilde{\theta}^{n}\right\|^{2}+\left\|\delta_{x} \widetilde{\theta}^{n-1}\right\|^{2}+\left\|\widetilde{c}^{n}\right\|^{2} \\
& \left.+\left\|\widetilde{\theta}^{n}\right\|^{2}+\left\|\hat{c}^{n}\right\|^{2}\right)-E_{c}\left(\tau^{2}+h^{2}\right)^{2},
\end{aligned}
$$

where we noted the fact that $\left(R_{w, 1 / 2}^{n+1}-R_{w, 1 / 2}^{n}\right) / 2 \tau \leq E_{c}\left(\tau^{2}+h^{2}\right)$ and $\left(R_{w, 1 / 2}^{n}-R_{w, 1 / 2}^{n-1}\right) / 2 \tau \leq E_{c}\left(\tau^{2}+h^{2}\right)$. Similarly we estimate $-\nabla_{t} \widetilde{\theta}_{M}^{n} \delta_{x} \widetilde{\theta}_{M-1 / 2}^{\hat{n}}$. From (47), we have

$$
\begin{aligned}
- & \delta_{x} \widetilde{\theta}_{M-1 / 2}^{\hat{n}} \\
= & \beta_{2} \widetilde{\theta}_{M}^{\widehat{n}} \\
& +\frac{h}{2 \kappa}\left[\left(c_{M}^{\widehat{n}}+\sigma\right) \nabla_{t} \widetilde{\theta}_{0}^{n}-u_{M-1 / 2}^{\widehat{n}} \delta_{x} \widetilde{\theta}_{M-1 / 2}^{\hat{n}}-\widetilde{R}_{\theta, M}^{n}\right] \\
& +R_{w, M-1 / 2}^{n},
\end{aligned}
$$

such that

$$
\begin{aligned}
& -\nabla_{t} \widetilde{\theta}_{M}^{n} \delta_{x} \widetilde{\theta}_{M-1 / 2}^{n} \geq \frac{\beta_{2}}{2} \nabla_{t}\left(\left(\widetilde{\theta}_{M}^{n}\right)^{2}\right)+\frac{h \sigma}{4 \kappa}\left|\nabla_{t} \widetilde{\theta}_{M}^{n}\right|^{2} \\
& +\nabla_{t}\left(R_{w, M-1 / 2}^{n} \widetilde{\theta}_{0}^{n}\right)-\frac{2 E_{3}^{2}}{\kappa \sigma}\left\|\delta_{x} \widetilde{\theta}^{\widehat{n}}\right\|^{2} \\
& -\frac{50 E_{3}^{2} \theta_{\max }^{2}}{\kappa \sigma}\left\|\delta_{x} \widehat{c}^{\hat{c}}\right\|^{2}-E_{c}\left(\tau^{2}+h^{2}\right)^{2}-E_{c}\left(\left\|\widetilde{\theta}^{n+1}\right\|^{2}\right. \\
& +\left\|\widetilde{\theta}^{n-1}\right\|^{2}+\left\|\delta_{x} \widetilde{\theta}^{n+1}\right\|^{2}+\left\|\delta_{x} \widetilde{\theta}^{n}\right\|^{2}+\left\|\delta_{x} \widetilde{\theta}^{n-1}\right\|^{2} \\
& \left.+\left\|\widetilde{c}^{n}\right\|^{2}+\left\|\widetilde{\theta}^{n}\right\|^{2}+\left\|\widetilde{c}^{\widehat{n}}\right\|^{2}\right) .
\end{aligned}
$$

For those terms in the right hand side, with (70), we have

$$
\begin{aligned}
& \left|J_{8}\right| \leq E_{c}\left\|\widehat{c^{n}}\right\|^{2}+\frac{\kappa}{16\left(c_{\max }+\sigma\right)}\left\|\delta_{x}^{2} \widetilde{\theta}^{\hat{n}}\right\|^{2}, \\
& \left|J_{9}\right| \leq \frac{E_{3}}{2\left(c_{\min }+\sigma\right)}\left|h \sum_{i=1}^{M-1}\left(\widetilde{u}_{i-1 / 2}^{\widehat{n}} \delta_{x}^{2} \widetilde{\theta}_{i}^{\widehat{n}}+\widetilde{u}_{i+1 / 2}^{\widehat{n}} \delta_{x}^{2} \widetilde{\theta}_{i}^{\hat{n}}\right)\right| \\
& \leq \frac{\kappa}{16\left(c_{\max }+\sigma\right)}\left\|\delta_{x}^{2} \widetilde{\theta}^{\hat{n}}\right\|^{2}+\frac{16 E_{3}^{2}\left(c_{\max }+\sigma\right)}{\left(c_{\min }+\sigma\right)^{2} \kappa} \\
& \cdot h \sum_{i=1}^{M-1}\left|\widetilde{u}_{i+1 / 2}^{\widehat{n}}\right|^{2} \leq \frac{\kappa}{16\left(c_{\max }+\sigma\right)}\left\|\delta_{x}^{2} \widetilde{\theta}^{\hat{n}}\right\|^{2} \\
& +\frac{320 E_{3}^{2}\left(c_{\max }+\sigma\right) \theta_{\max }^{2}}{\left(c_{\min }+\sigma\right)^{2} \kappa}\left\|\delta_{x} \hat{c^{\hat{c}}}\right\|^{2}+E_{c}\left(\left\|\delta_{x} \widetilde{\theta}^{n}\right\|^{2}\right. \\
& \left.+\left\|\widetilde{c}^{n}\right\|^{2}+\left\|\widetilde{\theta}^{n}\right\|^{2}\right)+E_{c}\left(\tau^{2}+h^{2}\right)^{2}, \\
& \left|J_{10}\right| \leq \frac{\kappa}{16\left(c_{\max }+\sigma\right)}\left\|\delta_{x}^{2} \widetilde{\theta}^{n}\right\|^{2}+E_{c}\left(\left\|\widetilde{c}^{n}\right\|^{2}+\left\|\widetilde{\theta}^{n}\right\|^{2}\right) \\
& +E_{c}\left(\tau^{2}+h^{2}\right)^{2} \text {, } \\
& \left|J_{11}\right| \leq E_{3} \mid h \sum_{i=1}^{M-1}\left(\delta_{x} \widetilde{\theta}_{i-1 / 2}^{\hat{n}} \delta_{x}^{2} \widetilde{\theta}_{i-1 / 2}^{\hat{n}}\right. \\
& \left.+\delta_{x} \widetilde{\theta}_{i+1 / 2}^{n} \delta_{x}^{2} \widetilde{\theta}_{i+1 / 2}^{\hat{n}}\right) \mid \\
& \leq 2 E_{3} \mid h \sum_{i=0}^{M-1}\left[\left(\sqrt{\frac{32 E_{3}\left(c_{\max }+\sigma\right)}{\kappa}} \delta_{x} \widetilde{\theta}_{i+1 / 2}^{n}\right)\right. \\
& \left.\cdot\left(\sqrt{\frac{\kappa}{32 E_{3}\left(c_{\max }+\sigma\right)}} \delta_{x}^{2} \widetilde{\theta}_{i+1 / 2}^{n}\right)\right] \mid \\
& \leq \frac{\kappa}{16\left(c_{\max }+\sigma\right)}\left\|\delta_{x}^{2} \widetilde{\theta}^{\hat{n}}\right\|^{2} \\
& +\frac{64 E_{3}^{2}\left(c_{\max }+\sigma\right)}{\kappa}\left\|\delta_{x} \widetilde{\theta}^{\widehat{n}}\right\|^{2} \text {. }
\end{aligned}
$$


Plugging the last six equations into (95), we get

$$
\begin{aligned}
& \frac{1}{2} \nabla_{t}\left\|\delta_{x} \widetilde{\theta}^{n}\right\|^{2}+\frac{\kappa}{4\left(c_{\max }+\sigma\right)}\left\|\delta_{x}^{2} \widetilde{\theta}^{n}\right\|^{2}+\frac{\beta_{1}}{2} \nabla_{t}\left(\left|\widetilde{\theta}_{0}^{n}\right|^{2}\right) \\
& +\frac{\beta_{2}}{2} \nabla_{t}\left(\left|\widetilde{\theta}_{M}^{n}\right|^{2}\right)+\nabla_{t}\left(R_{w, M-1 / 2}^{n} \widetilde{\theta}_{M}^{n}-R_{w, 1 / 2}^{n} \widetilde{\theta}_{0}^{n}\right) \\
& \leq E_{7}\left\|\delta_{x} \widetilde{c}^{n}\right\|^{2}+E_{8}\left\|\delta_{x} \widetilde{\theta}^{n}\right\|^{2}+E_{c}\left(\tau^{2}+h^{2}\right)^{2} \\
& +E_{c}\left(\left\|\delta_{x} \widetilde{\theta}^{n}\right\|^{2}+\left\|\widetilde{c}^{n}\right\|^{2}+\left\|\widetilde{c}^{\hat{n}}\right\|^{2}+\left\|\widetilde{\theta}^{n}\right\|^{2}+\left\|\widetilde{\theta}^{n+1}\right\|^{2}\right. \\
& \left.+\left\|\widetilde{\theta}^{n-1}\right\|^{2}+\left\|\delta_{x} \widetilde{\theta}^{n+1}\right\|^{2}+\left\|\delta_{x} \widetilde{\theta}^{n-1}\right\|^{2}\right) .
\end{aligned}
$$

Multiplying the last equation with $0 \leq s_{2} \leq E_{9}$ and adding it into (90), we have

$$
\begin{aligned}
& \frac{s_{2}}{2} \nabla_{t}\left\|\delta_{x} \widetilde{\theta}^{n}\right\|^{2}+\frac{s_{2} \kappa}{4\left(c_{\max }+\sigma\right)}\left\|\delta_{x}^{2} \widetilde{\theta}^{n}\right\|^{2}+\frac{\theta_{\min }}{16}\left\|\delta_{x} \widehat{c}^{\hat{n}}\right\|^{2} \\
& +\frac{\kappa s_{1}}{8}\left\|\delta_{x} \widetilde{\theta}^{\widehat{n}}\right\|^{2}+\nabla_{t}\left\|\widetilde{c}^{n}\right\|^{2}+\frac{\beta_{1} s_{2}}{2} \nabla_{t}\left(\left|\widetilde{\theta}_{0}^{n}\right|^{2}\right) \\
& +\frac{\beta_{2} s_{2}}{2} \nabla_{t}\left(\left|\widetilde{\theta}_{M}^{n}\right|^{2}\right)+s_{2} \nabla_{t}\left(-R_{w, 1 / 2}^{n} \widetilde{\theta}_{0}^{n}\right. \\
& \left.+R_{w, M-1 / 2}^{n} \widetilde{\theta}_{M}^{n}\right)+\frac{s_{1} h}{2}\left[\frac{1}{2} \nabla_{t}\left[\left(c_{0}^{n}+\sigma\right)\left|\widetilde{\theta}_{0}^{n}\right|^{2}\right]\right. \\
& \left.+\sum_{i=1}^{M-1} \nabla_{t}\left[\left(c_{i}^{n}+\sigma\right)\left|\widetilde{\theta}_{i}^{n}\right|^{2}\right]+\frac{1}{2} \nabla_{t}\left[\left(c_{M}^{n}+\sigma\right)\left|\widetilde{\theta}_{M}^{n}\right|^{2}\right]\right] \\
& \quad \leq E_{c}\left(\left\|\delta_{x} \widetilde{\theta}^{n+1}\right\|^{2}+\left\|\widetilde{c}^{n+1}\right\|^{2}+\left\|\widetilde{\theta}^{n+1}\right\|^{2}\right) \\
& +E_{c}\left(\left\|\delta_{x} \widetilde{\theta}^{n}\right\|^{2}+\left\|\delta_{x} \widetilde{\theta}^{n-1}\right\|^{2}+\left\|\widetilde{c}^{n}\right\|^{2}+\left\|\widetilde{c}^{n-1}\right\|^{2}\right. \\
& \left.+\left\|\widetilde{\theta}^{n}\right\|^{2}+\left\|\widetilde{\theta}^{n-1}\right\|^{2}\right) .+E_{c}\left(\tau^{2}+h^{2}\right)^{2} .
\end{aligned}
$$

Letting $s=\min \left(s_{2} / 2, s_{2} \kappa / 2\left(c_{\max }+\sigma\right), \theta_{\min } / 8, \kappa s_{1} / 4,1\right.$, $\left.s_{1}, s_{2} \beta_{1} / 2, s_{2} \beta_{2} / 2\right)$, we get

$$
\begin{aligned}
& s \nabla_{t}\left\|\delta_{x} \widetilde{\theta}^{n}\right\|^{2}+\frac{s}{2}\left\|\delta_{x}^{2} \widetilde{\theta}^{n}\right\|^{2}+\frac{s}{2}\left\|\delta_{x} \widehat{c}^{\hat{c}}\right\|^{2}+\frac{s}{2}\left\|\delta_{x} \widetilde{\theta}^{n}\right\|^{2} \\
& +s \nabla_{t}\left\|\widetilde{c}^{n}\right\|^{2}+s \nabla_{t}\left(\left|\widetilde{\theta}_{0}^{n}\right|^{2}\right)+s \nabla_{t}\left(\left|\widetilde{\theta}_{M}^{n}\right|^{2}\right) \\
& +s \nabla_{t}\left(-R_{w, 1 / 2}^{n} \widetilde{\theta}_{0}^{n}+R_{w, M-1 / 2}^{n} \widetilde{\theta}_{M}^{n}\right) \\
& +\frac{s h}{2}\left[\frac{1}{2} \nabla_{t}\left[\left(c_{0}^{n}+\sigma\right)\left|\widetilde{\theta}_{0}^{n}\right|^{2}\right]\right. \\
& +\sum_{i=1}^{M-1} \nabla_{t}\left[\left(c_{i}^{n}+\sigma\right)\left|\widetilde{\theta}_{i}^{n}\right|^{2}\right] \\
& +\frac{1}{2} \nabla_{t}\left[\left(c_{M}^{n}+\sigma\right)\left|\widetilde{\theta}_{M}^{n}\right|^{2}\right] \leq E_{c}\left(\left\|\delta_{x} \widetilde{\theta}^{n+1}\right\|^{2}\right. \\
& +\left\|\delta_{x} \widetilde{\theta}^{n}\right\|^{2}+\left\|\delta_{x} \widetilde{\theta}^{n-1}\right\|^{2}+\left\|\widetilde{c}^{n+1}\right\|^{2}+\left\|\widetilde{c}^{n}\right\|^{2}
\end{aligned}
$$

$$
\begin{aligned}
& \left.+\left\|\widetilde{c}^{n-1}\right\|^{2}+\left\|\widetilde{\theta}^{n+1}\right\|^{2}+\left\|\widetilde{\theta}^{n}\right\|^{2}+\left\|\widetilde{\theta}^{n-1}\right\|^{2}\right) \\
& +E_{c}\left(\left|\widetilde{\theta}_{0}^{n+1}\right|^{2}+\left|\widetilde{\theta}_{0}^{n}\right|^{2}+\left|\widetilde{\theta}_{0}^{n-1}\right|^{2}+\left|\widetilde{\theta}_{M}^{n+1}\right|^{2}+\left|\widetilde{\theta}_{M}^{n}\right|^{2}\right. \\
& \left.+\left|\widetilde{\theta}_{M}^{n-1}\right|^{2}\right)+E_{c}\left(\tau^{2}+h^{2}\right)^{2} .
\end{aligned}
$$

Letting

$$
\begin{aligned}
F_{n+1} & =s\left\|\delta_{x} \widetilde{\theta}^{n+1}\right\|^{2}+s\left\|\widetilde{c}^{n+1}\right\|^{2}+\left|\widetilde{\theta}_{0}^{n}\right|^{2}+\left|\widetilde{\theta}_{M}^{n}\right|^{2} \\
+ & \frac{s h}{2}\left(\frac{1}{2}\left(c_{0}^{n+1}+\sigma\right)\left|\widetilde{\theta}_{0}^{n+1}\right|^{2}\right. \\
+ & \sum_{i=1}^{M-1}\left(\left(c_{i}^{n+1}+\sigma\right)\left|\widetilde{\theta}_{i}^{n+1}\right|^{2}\right) \\
+ & \left.\frac{1}{2}\left(c_{M}^{n+1}+\sigma\right)\left|\widetilde{\theta}_{M}^{n+1}\right|^{2}\right),
\end{aligned}
$$

we have

$$
\begin{gathered}
F_{n+1}-F_{n-1}+s \tau\left(\left\|\delta_{x} \widetilde{c}^{\hat{n}}\right\|^{2}+\left\|\delta_{x} \widetilde{\theta}^{\hat{n}}\right\|^{2}+\left\|\delta_{x}^{2} \widetilde{\theta}^{\hat{n}}\right\|^{2}\right) \\
\leq 2 \tau E_{c}\left(F_{n+1}+F_{n}+F_{n-1}\right)+2 \tau E_{c}\left(\tau^{2}+h^{2}\right)^{2},
\end{gathered}
$$

from which we can find $\alpha=\left(-\tau E_{c}+\sqrt{1-3\left(\tau E_{c}\right)^{2}}\right) /\left(1-2 \tau E_{c}\right)$ and $\lambda=\left(\tau E_{c}+\sqrt{1-3\left(\tau E_{c}\right)^{2}}\right) /\left(1-2 \tau E_{c}\right)$, such that

$$
\begin{aligned}
F_{n+1} & +\alpha F_{n} \\
& +\frac{s \tau}{1-2 \tau E_{c}}\left(\left\|\delta_{x} \widehat{c^{n}}\right\|^{2}+\left\|\delta_{x} \widetilde{\theta}^{\hat{n}}\right\|^{2}+\left\|\delta_{x}^{2} \widetilde{\theta}^{\widehat{n}}\right\|^{2}\right) \\
\leq & \lambda\left(F_{n}+\alpha F_{n-1}\right)+\frac{2 \tau E_{c}}{1-2 \tau E_{c}}\left(\tau^{2}+h^{2}\right)^{2} .
\end{aligned}
$$

When $\tau E_{c}<1 / 4$,

$$
\begin{aligned}
& F_{n+1}+\alpha F_{n}+\sum_{m=1}^{n} \tau s\left(\left\|\delta_{x} \widehat{c}^{\widehat{m}}\right\|^{2}+\left\|\delta_{x} \widetilde{\theta}^{\widehat{m}}\right\|^{2}+\left\|\delta_{x}^{2} \widehat{\theta}^{\widehat{n}}\right\|^{2}\right) \\
& \leq e^{6 E_{c} \mathscr{T}}\left(F_{1}+F_{0}+\left(\tau^{2}+h^{2}\right)^{2}\right),
\end{aligned}
$$

and

$$
\begin{aligned}
& s\left\|\delta_{x} \widetilde{\theta}^{n+1}\right\|^{2}+s\left\|\widetilde{c}^{n+1}\right\|^{2}+\frac{s h}{2}\left(\frac{1}{2}\left(c_{0}^{n+1}+\sigma\right)\left|\widetilde{\theta}_{0}^{n+1}\right|^{2}\right. \\
& \left.+\sum_{i=1}^{M-1}\left(c_{i}^{n+1}+\sigma\right)\left|\widetilde{\theta}_{i}^{n+1}\right|^{2}+\frac{1}{2}\left(c_{M}^{n+1}+\sigma\right)\left|\widetilde{\theta}_{M}^{n+1}\right|^{2}\right) \\
& +\sum_{m=1}^{n} \tau s\left(\left\|\delta_{x} \widehat{\widetilde{c}} \widehat{m}\right\|^{2}+\left\|\delta_{x} \widetilde{\theta}^{\widehat{m}}\right\|^{2}+\left\|\delta_{x}^{2} \widetilde{\theta}^{\hat{n}}\right\|^{2}\right) \\
& \leq e^{6 E_{c} \mathscr{T}}\left(\tau^{2}+h^{2}\right)^{2} .
\end{aligned}
$$


TABLE 1: Numerical results of Example 1 with $\tau=h$.

\begin{tabular}{lcccc}
\hline & & $\left\|\widetilde{c}^{\hat{n}}\right\|$ & & \\
$t$ & $h=L / 200$ & $h=L / 400$ & Order $r$ \\
\hline$t=0.25$ & $2.914 \mathrm{E}-03$ & $1.004 \mathrm{E}-03$ & $0.254 \mathrm{E}-03$ & 1.99 \\
$t=0.50$ & $8.163 \mathrm{E}-03$ & $2.178 \mathrm{E}-03$ & $0.527 \mathrm{E}-03$ & 2.03 \\
$t=0.75$ & $1.397 \mathrm{E}-02$ & $0.350 \mathrm{E}-02$ & $0.084 \mathrm{E}-02$ & 2.04 \\
$t=1.00$ & $2.032 \mathrm{E}-02$ & $0.496 \mathrm{E}-02$ & $0.118 \mathrm{E}-02$ & 2.05 \\
\hline & $\left\|\widetilde{\theta}^{n}\right\|$ & & Order $r$ \\
$t$ & $h=L / 200$ & $h=L / 400$ & $h=L / 800$ & 2.00 \\
\hline$t=0.25$ & $9.509 \mathrm{E}-03$ & $3.795 \mathrm{E}-03$ & $0.947 \mathrm{E}-03$ & 2.03 \\
$t=0.50$ & $3.860 \mathrm{E}-03$ & $1.042 \mathrm{E}-03$ & $0.253 \mathrm{E}-03$ & 2.02 \\
$t=0.75$ & $7.115 \mathrm{E}-02$ & $1.799 \mathrm{E}-02$ & $0.439 \mathrm{E}-02$ & 2.02 \\
$t=1.00$ & $1.069 \mathrm{E}-01$ & $0.265 \mathrm{E}-01$ & $0.065 \mathrm{E}-01$ & \\
\hline
\end{tabular}

Since $E_{c}$ is independent of $E_{0}$, with

$$
E_{0}=\frac{e^{6 E_{c} \mathscr{T}}}{s},
$$

we find that (30) holds for $k=n$. The induction and the proof of the theorem are completed.

\section{Numerical Examples}

We now numerically evaluate the performance of the proposed leap-frog scheme.

Example 1. First, we test the accuracy of our algorithm in an artificial example which is taken from [23]. The system is

$$
\begin{gathered}
C_{t}+\left(u_{g} C\right)_{x}=-\Gamma+f_{C}(x, t), \\
(C+\sigma) T_{t}-\left(\kappa T_{x}\right)_{x}+u_{g} C_{x} T_{x} \\
=[\lambda+\sigma T] \Gamma+f_{T}(x, t),
\end{gathered}
$$

with the boundary conditions (8)-(12), where $f_{C}, f_{T}, \mu_{i}$, and $v_{i}$ are coefficients decided by the exact solution

$$
\begin{aligned}
& C(x, t)=e^{-0.72 t} x^{2}(1-x)^{2}+a(1-x)+b x, \\
& T(x, t)=50 e^{-0.72 t} x^{2}(1-x)^{2}+c(1-x)+d x,
\end{aligned}
$$

with $a, b, c, d$ being constants.

We apply the uncoupled leap-frog finite difference method to solve the artificial example. We choose $\mathscr{T}=1$ and $L=1$. Since the proposed scheme is of the second order in both spatial and temporal directions, we take $\tau=h$ such that the error bound is proportional to $h^{2}$. We present the $L^{2}$-norm errors and the order of convergence $h^{r}$ in Table 1 with $h=L / 200, L / 400, L / 800$ at different time level. We can see clearly from Table 1 that the $L^{2}$-norm errors for both components are proportional to $h^{2}$, which confirms our theoretical analysis.

Example 2. In the second example, we discuss a typical clothing assembly in the textile industry $[2,4,25]$. The
TABLE 2: Physical parameters for batting materials.

\begin{tabular}{lcc}
\hline parameter & polyester & unit \\
\hline$\rho_{f}$ & $1.39 \times 10^{3}$ & $\mathrm{kgm}^{-3}$ \\
$\rho_{w}$ & $1 \times 10^{3}$ & $\mathrm{kgm}^{-3}$ \\
$\epsilon^{\prime}$ & 0.993 & \\
$\kappa_{f}$ & $1 \times 10^{-1}$ & $\mathrm{~W} \mathrm{~m}{ }^{-1} \mathrm{~K}^{-1}$ \\
$\kappa_{w}$ & $5.7 \times 10^{-1}$ & $\mathrm{~W} \mathrm{~m}^{-1} \mathrm{~K}^{-1}$ \\
$C_{v f}$ & $1.17 \times 10^{6}$ & $\mathrm{~J} \mathrm{~m}^{-1} \mathrm{~K}^{-1}$ \\
$L$ & $4.92 \times 10^{-2}$ & $\mathrm{~m}$ \\
\hline
\end{tabular}

clothing assembly consists of three layers, in the middle is porous fibrous media, and the outside cover is exposed to a cold environment with fixed temperature and relative humidity while the inside cover is exposed to a mixture of air and vapor at higher temperature and relative humidity. In this paper, polyester porous media with laminated or nylon cover materials are tested. To compare with the experimental data in [12], a water equation is added to equations (1)-(2):

$$
\frac{\partial}{\partial t}\left(\rho_{w}\left(1-\epsilon^{\prime}\right) W\right)=M_{w} \Gamma_{c e}
$$

where $W$ is water content, $\rho_{w}$ is the density of water, $\epsilon$ is the porosity with liquid water content, and $\epsilon^{\prime}$ is the porosity without liquid water content. We have

$$
\epsilon=\epsilon^{\prime}-\frac{\rho_{f}}{\rho_{w}} W\left(1-\epsilon^{\prime}\right)
$$

and the effective heat conductivity is defined by

$$
\kappa=\epsilon \kappa_{g}+(1-\epsilon) \kappa_{s}
$$

where $\kappa_{g}$ is the thermal conductivity of gas and $\kappa_{s}$ is the thermal conductivity of the fiber-water mixture $[2,6,7]$, given by

$$
\kappa_{s}=\left(\frac{1}{\rho_{f}}+\frac{W}{\rho_{w}}\right)\left(\frac{1}{\rho_{f} \kappa_{f}}+\frac{W}{\rho_{w} \kappa_{w}}\right)^{-1} .
$$

The values of these physical parameters for polyester media are presented in Table 2. Other parameters values can be found in $[2,6,7]$. 

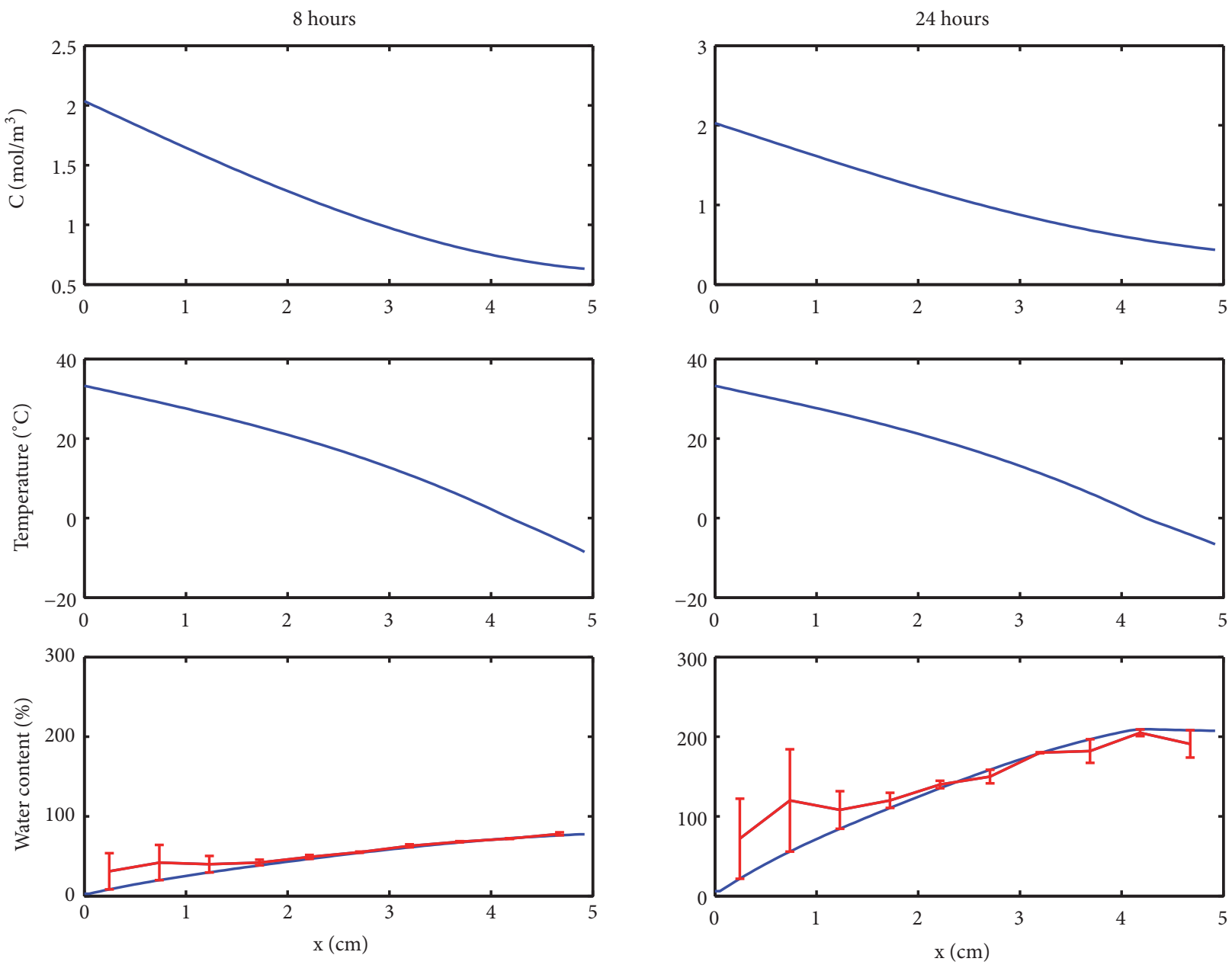

FiguRE 1: Numerical results for 10-pile polyester batting sandwiched by two layers of laminated fabric.

The initial conditions for the vapor, temperature, and water content are given by

$$
C=65 \% \frac{P_{s a t}(T)}{R T}, \quad T=25^{\circ} C, W=0 \text {, at } t=0 .
$$

We apply the uncoupled leap-frog finite difference method for solving the sweat transport system defined in (1)(2) coupled with the water equation (115). Since only the right side of the water equation includes $c$ and $\theta$, therefore, the water equation is calculated separately. Numerically, at each time step, we first find solution $c_{j}^{n+1}, \theta_{j}^{n+1}$ by procedure (18)(26), and then $W_{j}^{n+1}$ can be solved by following nonnormalized discrete formate:

$$
\frac{\rho_{f}\left(1-\epsilon_{j}^{\prime n}\right)}{\tau}\left(W_{j}^{n+1}-W_{j}^{n}\right)=M_{w} \Gamma_{\mathrm{ce}, j}^{n} .
$$

Then we evaluate the parameters explicitly in (18)-(26) based on $W_{j}^{n+1}$. Here all numerical results are obtained by taking the time step size $\tau=20 \mathrm{~s}$ and spatial mesh size $h=$ $L / 100$. We present numerical results of vapor, temperature, and water content at 8 hours and 24 hours, respectively, for the porous polyester media assembly with laminated cover in Figure 1 and with nylon cover in Figure 2. The comparisons between numerical results of water content and experimental measurements [12] are given in last two subfigures, where the blue lines represent the numerical solution and the red line is given by experimental measurement.

\section{Conclusion}

As a subsequent work of [23], we have presented an uncoupled leap-frog finite difference method for the sweat transport system in porous textile media, which is governed by a strongly coupled, nonlinear parabolic system. Optimal $L^{2}$ error estimates were presented, which imply that the numerical scheme is unconditionally stable. Both theoretical analysis and numerical example indicate that the current scheme is second order accurate in both the temporal and spatial directions. Since the scheme is decoupled for the system, the method can be applied efficiently for problems in higherdimensional space. Under certain time-step restrictions, the analysis can also be extended to the multidimensional problems. 

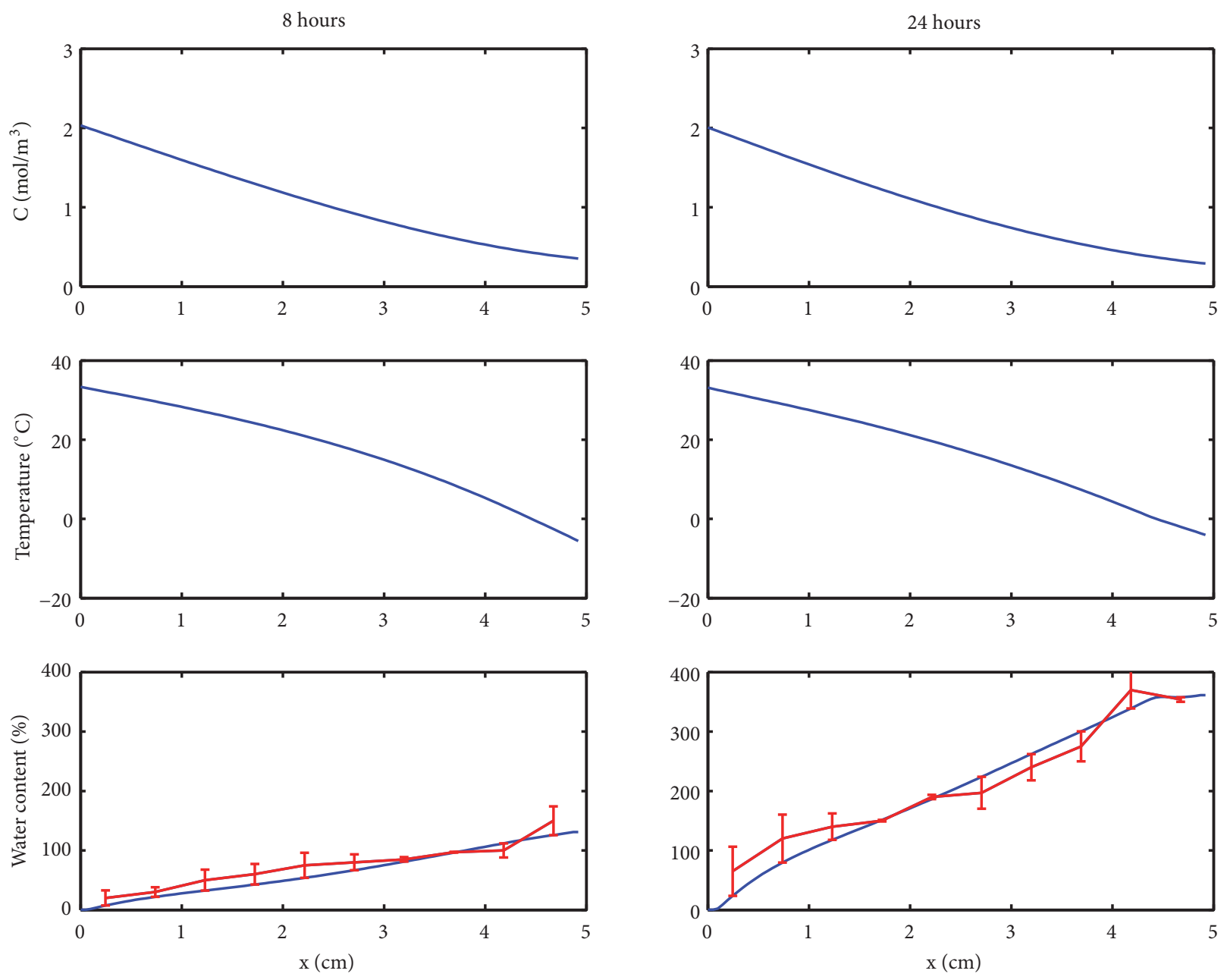

FIGURE 2: Numerical results for 10-pile polyester batting sandwiched by two layers of nylon fabric.

\section{Data Availability}

No data were used to support this study.

\section{Conflicts of Interest}

The authors declare that they have no conflicts of interest.

\section{Acknowledgments}

The authors wish to thank Professors Z. Sun and W. Sun for helpful discussions. This research was partially supported by National Natural Science Foundation of China (Nos. 11601346, 11501377), Shenzhen Key Laboratory of Advanced Machine Learning and Applications, Guangdong Key Laboratory of Intelligent Information Processing, and Interdisciplinary Innovation Team of Shenzhen University.

\section{References}

[1] M. K. Choudhary, K. C. Karki, and S. V. Patankar, "Mathematical modeling of heat transfer, condensation, and capillary flow in porous insulation on a cold pipe," International Journal of Heat and Mass Transfer, vol. 47, pp. 5629-5638, 2004.
[2] J. Fan, X. Cheng, X. Wen, and W. Sun, "An improved model of heat and moisture transfer with phase change and mobile condensates in fibrous insulation and comparison with experimental results," International Journal of Heat and Mass Transfer, vol. 47, no. 10-11, pp. 2343-2352, 2004.

[3] C. V. le, N. G. ly, and R. Postle, "Heat and Moisture Transfer in Textile Assemblies: Part I: Steaming of Wool, Cotton, Nylon, and Polyester Fabric Beds," Textile Research Journal, vol. 65, no. 4, pp. 203-212, 1995.

[4] Y. li and Q. Zhu, "Simultaneous Heat and Moisture Transfer with Moisture Sorption, Condensation, and Capillary Liquid Diffusion in Porous Textiles," Textile Research Journal, vol. 73, no. 6, pp. 515-524, 2003.

[5] P. W. Gibson and M. Charmchi, "Modeling convection/diffusion processes in porous textiles with inclusion of humiditydependent air permeability," International Communications in Heat and Mass Transfer, vol. 24, no. 5, pp. 709-724, 1997.

[6] H. Huang, C. Ye, and W. Sun, "Moisture transport in fibrous clothing assemblies," Journal of Engineering Mathematics, vol. 61, no. 1, pp. 35-54, 2008.

[7] C. Ye, H. Huang, J. Fan, and W. Sun, "Numerical study of heat and moisture transfer in textile materials by a finite volume method," Communications in Computational Physics, vol. 4, no. 4, pp. 929-948, 2008. 
[8] Q. Zhang, B. Li, and W. Sun, "Heat and sweat transport through clothing assemblies with phase changes, condensation/evaporation and absorption," Proceedings of the Royal Society A Mathematical, Physical and Engineering Sciences, vol. 467, no. 2136, pp. 3469-3489, 2011.

[9] Q. Zhang, "Mathematical modeling and numerical study of carbonation in porous concrete materials," Applied Mathematics and Computation, vol. 281, pp. 16-27, 2016.

[10] C. Ye, B. Li, and W. Sun, "Quasi-steady-state and steady-state models for heat and moisture transport in textile assemblies," Proceedings of the Royal Society A Mathematical, Physical and Engineering Sciences, vol. 466, no. 2122, pp. 2875-2896, 2010.

[11] F. E. Jones, Evaporation of Water, Lewis Publishers Inc, Michigan, Mich, USA, 1992.

[12] J. Fan, X. Cheng, and Y. S. Chen, "An experimental investigation of moisture absoption and condensation in fibrous insulations under low temperature," Experimental Thermal and Fluid Science, vol. 27, pp. 723-729, 2002.

[13] W. Dai and G. Li, "A finite difference scheme for solving parabolic two-step micro-heat transport equations in a doublelayered micro-sphere heated by ultrashort-pulsed lasers," Numerical Methods for Partial Differential Equations, vol. 22, no. 6, pp. 1396-1417, 2006.

[14] R. Eymard, J. Fuhrmann, and K. Gartner, "A finite volume scheme for nonlinear parabolic equations derived from onedimensional local Dirichlet problems," Numerische Mathematik, vol. 102, pp. 463-495, 2006.

[15] R. Eymard, T. Gallouët, R. Herbin, and A. Michel, "Convergence of a finite volume scheme for nonlinear degenerate parabolic equations," Numerische Mathematik, vol. 92, pp. 41-82, 2002.

[16] C. V. Pao, "Numerical analysis of coupled systems of nonlinear parabolic equations," SIAM Journal on Numerical Analysis, vol. 36, no. 2, pp. 393-416, 1999.

[17] H. J. Schroll, "Convergence of implicit finite difference methods applied to nonlinear mixed systems," SIAM Journal on Numerical Analysis, vol. 33, no. 3, pp. 997-1013, 1996.

[18] W. Sun and G. Yuan, "Stability condition for difference schemes for parabolic systems," SIAM Journal on Numerical Analysis, vol. 38 , no. 2, pp. 548-555, 2000.

[19] H. Wang, "An optimal-order error esti mate for a family of ELLAM-MFEM approximations to porous medium flow," SIAM Journal on Numerical Analysis, vol. 46, no. 4, pp. 21332152, 2008.

[20] Y. Yuan, "The upwind finite difference fractional steps methods for two-phase compressible flow in porous media," Numerical Methods for Partial Differential Equations, vol. 19, no. 1, pp. 6788, 2003.

[21] B. Li, W. Sun, and Y. Wang, "Global existence of weak solution to the heat and moisture transport system in fibrous porous media," Journal of Differential Equations, vol. 249, no. 10, pp. 2618-2642, 2010.

[22] B. Li and W. Sun, "Global existence of weak solution for nonisothermal multicomponent flow in porous textile media," SIAM Journal on Mathematical Analysis, vol. 42, no. 6, pp. 30763102, 2010.

[23] W. Sun and Z. Sun, "Finite difference methods for a nonlinear and strongly coupled heat and moisture transport system in textile materials," Numerische Mathematik, 2011.

[24] A. A. Samarskii and B. B. Andreev, Finite Difference Methods for Elliptic Equation, Nauka, Moscow, Russia, 1976.
[25] J. Fan, Z. Luo, and Y. Li, "Heat and moisture transfer with sorption and condensation in porous clothing assemblies and numerical simulation," International Journal of Heat and Mass Transfer, vol. 43, pp. 2989-3000, 2000. 


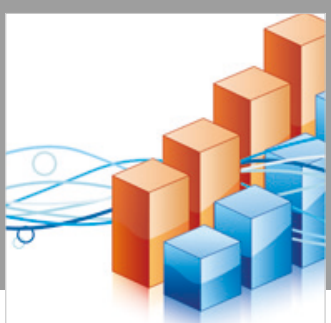

Advances in

Operations Research

\section{-n-m}
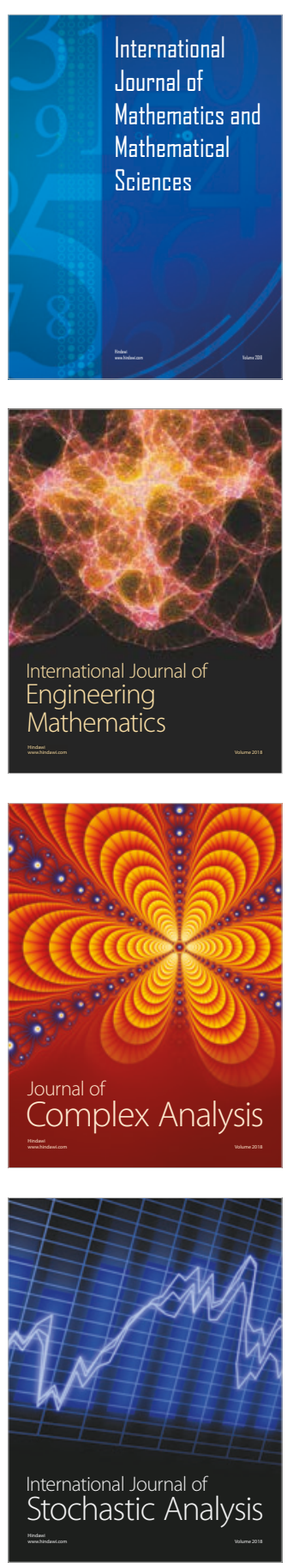
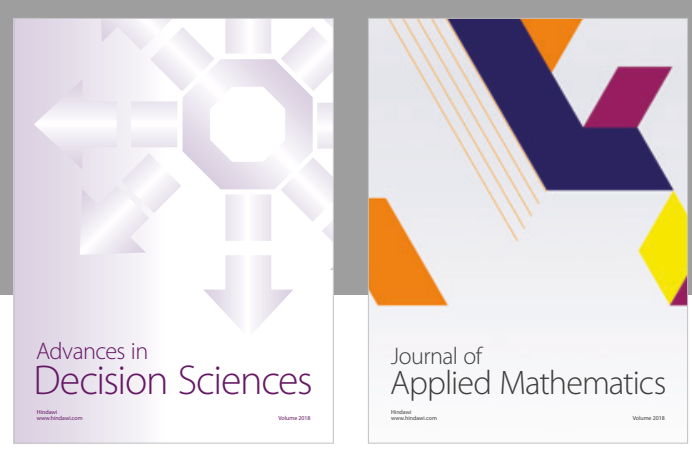

Journal of

Applied Mathematics
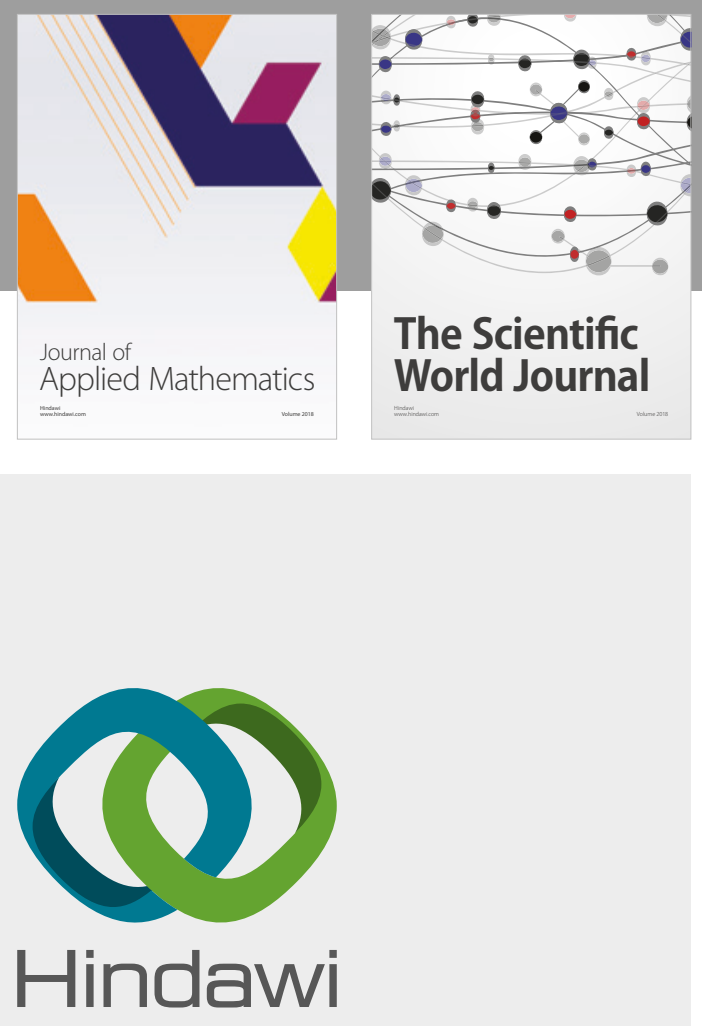

Submit your manuscripts at

www.hindawi.com

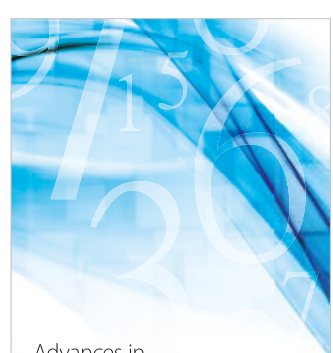

Advances in
Numerical Analysis
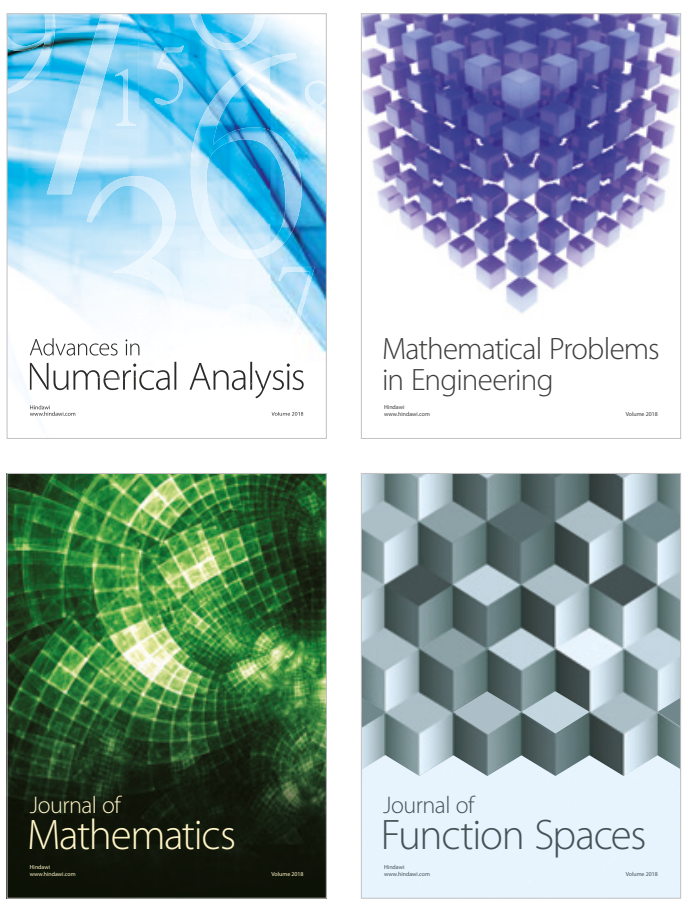

Mathematical Problems in Engineering

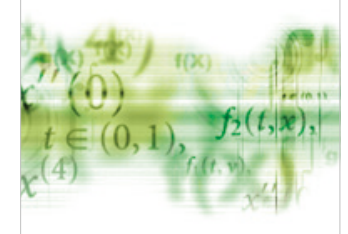

International Journal of

Differential Equations

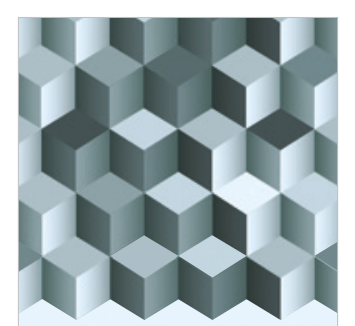

Journal of

Function Spaces

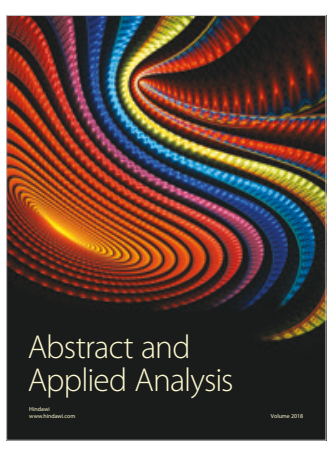

The Scientific

World Journal

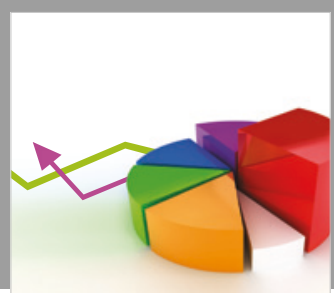

Journal of

Probability and Statistics
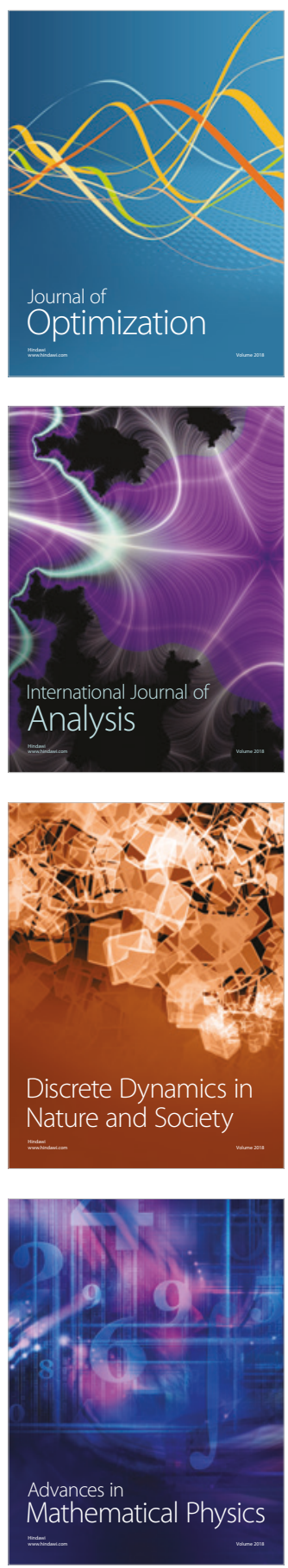